\title{
Clinical use of electronic portal imaging: Report of AAPM Radiation Therapy Committee Task Group 58
}

\author{
Michael G. Herman ${ }^{\text {a) }}$ \\ Division of Radiation Oncology, Mayo Clinic, Rochester, Minnesota 55905 \\ James M. Balter \\ Radiation Oncology Department, University of Michigan, Ann Arbor, Michigan 48109 \\ David A. Jaffray \\ Radiation Oncology Department, William Beaumont Hospital, Royal Oak, Michigan 48073 \\ Kiarin P. McGee \\ Department of Radiology, Mayo Clinic, Rochester, Minnesota 55902 \\ Peter Munro \\ Physics Department, London Regional Cancer Centre, London Ontario N6A 4L6, Canada \\ Shlomo Shalev \\ Masthead Imaging, Nanaimo, British Columbia V9R 2R2, Canada \\ Marcel Van Herk \\ Radiotherapy Department, Netherlands Cancer Institute, Amsterdam, The Netherlands \\ John W. Wong \\ Radiation Oncology Department, William Beaumont Hospital, Royal Oak, Michigan 48073
}

(Received 19 January 2001; accepted for publication 1 March 2001)

\begin{abstract}
AAPM Task Group 58 was created to provide materials to help the medical physicist and colleagues succeed in the clinical implementation of electronic portal imaging devices (EPIDs) in radiation oncology. This complex technology has matured over the past decade and is capable of being integrated into routine practice. However, the difficulties encountered during the specification, installation, and implementation process can be overwhelming. TG58 was charged with providing sufficient information to allow the users to overcome these difficulties and put EPIDs into routine clinical practice. In answering the charge, this report provides; comprehensive information about the physics and technology of currently available EPID systems; a detailed discussion of the steps required for successful clinical implementation, based on accumulated experience; a review of software tools available and clinical use protocols to enhance EPID utilization; and specific quality assurance requirements for initial and continuing clinical use of the systems. Specific recommendations are summarized to assist the reader with successful implementation and continuing use of an EPID. (c) 2001 American Association of Physicists in Medicine. [DOI: 10.1118/1.1368128]
\end{abstract}

Key words: electronic portal imaging, portal imaging, radiation therapy imaging

\section{TABLE OF CONTENTS}

I. INTRODUCTION.................. 713

II. THE PHYSICS OF PORTAL IMAGING. ...... 714

A. Contrast........................ 714

B. Signal-to-noise ratio.................. 714

1. Quantum noise................. 714

2. Quantum efficiency.............. 715

3. Other sources of noise............. 716

C. Spatial resolution.................... 717

D. X-ray scatter................... 717

III. THE TECHNOLOGY OF MEGAVOLTAGE

IMAGING. . . . . . . . . . . . . . . . . . . . 717

A. Matrix ion chamber................. 718

B. Camera-based EPIDs. .................. 719

IV. COMMISSIONING AND QUALITY

ASSURANCE FOR EPIDs.
A. Installation and commissioning. .......... 721

1. Dose control.................... 721

2. Calibration................... 722

3. Linearity.................... 722

4. Image quality................ 722

5. Software........................ 723

B. Quality assurance................... 723

V. CLINICAL APPLICATION OF EPIDs......... 727

A. Preparing for EPID implementation........ 727

B. Software tools...................... 727

1. Image acquisition. . . . . . . . . . . . . 727

a. Single exposure (localization)........ 727

b. Verification image............... 727

c. Double exposure................ 727

d. Movie loops.................... 727

2. Image enhancement tools........... 727

3. Setup verification/error detection tools.... 727 
C. EPID clinical use.................. 730

1. EPID clinical protocol (step by step)..... 730

2. On-line EPID use............... 730

3. Off-line EPID use............... 731

a. Simple off-line. . . . . . . . . . . . . . 731

b. Monitoring. . . . . . . . . . . 731

c. Statistical models/decision rules....... 731

D. Advanced applications.................. 732

1. Treatment QA................... 732

2. Exit dosimetry............... 732

VI. COST AND FUTURE.................. 733

A. Cost......................... 733

B. Future..................... 734

VII. SUMMARY AND RECOMMENDATIONS.... 734

ACKNOWLEDGMENTS ................... 734

\section{INTRODUCTION}

A critical requirement in radiation therapy is accurate dayto-day treatment setup. Early studies based on port films indicated the benefits of portal verification. ${ }^{1-4}$ Numerous subsequent studies have characterized the magnitude and nature of setup errors for a variety of clinical conditions. Random and systematic errors of up to $6 \mathrm{~mm}(\sigma)$ have been reported in previous studies. ${ }^{2,3,5-24}$

An effective means to reduce setup error would be to increase the frequency of treatment verification with portal imaging. ${ }^{25}$ Such action using port film is time consuming and labor intensive and can reduce throughput in a busy radiation therapy department. In addition, quantitative interpretation of geometric discrepancies is difficult and tedious to perform with nondigital imaging systems. ${ }^{26}$ The need for an improved portal imaging system to enhance verification of conformal radiation therapy spurred the development of on-line electronic portal imaging devices (EPIDs).

The modern era of electronic portal imaging began in the early 1980s with demonstration by the late Norman Baily of the use of a fluoroscopic system to acquire megavoltage transmission images. ${ }^{27}$ The introduction of the scanning liquid ionization chamber system in 1990 was quickly followed by the introduction of camera-based fluoroscopic EPIDs from other manufacturers. At present, EPIDs are commercially available in the US from at least five vendors. Initially, these devices were embraced with great expectation by the radiation therapy community. At the time when Task Group 58 (TG58) was formed in 1995, about 250 systems had been sold in the US. In years since, informal surveys indicate that the initial promise has not led to widespread clinical application of EPIDs. An informal survey of 69 institutions with EPIDs, conducted by members of TG58, indicated that 25\% do not use the devices at all. The most common mode of operation is for the radiotherapists to perform visual inspection of the patient setup as a first line of action to reduce large setup errors or mistakes. Only 50\% of the surveyed institutions have secondary review stations and only half of these appear to have comprehensive analysis tools. About $40 \%$ of the institutions with EPIDs have developed a comprehensive quality assurance (QA) program, but fewer than half of these perform the program regularly. Thirty-five percent of respondents do not have a QA program at all. The majority of users surveyed consider image quality from current EPIDs inferior to that of port films and thus the EPID is not used, contrary to statements of superior EPID resolution repeatedly made in the literature. On the other hand, EPIDs are used because many users believe that these devices save time and provide quantitative feedback.

It is clear that EPID technology is underutilized in the US. Furthermore, EPIDs are not used to produce their intended clinical benefit. Despite the impressive clinical results of $\mathrm{Eu}-$ ropean studies, ${ }^{28,29}$ it remains clear that apparent hurdles limit EPID utilization in the US. TG58 was formed to help AAPM members understand and implement EPID technology. It is the goal of this report to provide information to enhance and encourage effective use of these powerful devices.

The specific charges of Task Group 58 are as follows.

(1) To provide comprehensive technical information about the operation, limitations, and system characteristics of the various commercially available EPIDs for the purpose of implementation, use, and developing quality assurance programs.

(2) To summarize existing experience on the effective implementation and use of the EPID for imaging in various clinical treatment sites and conditions from simple film replacement to quantitative statistical methods.

(3) To describe tools currently available for on-line and offline evaluations of the images.

(4) To specify the requirements and discuss issues related to quality assurance for EPID systems, including the archive and management of the large amount of imaging data.

Aside from Sec. I, the TG58 report consists of five major sections and a summary. Section II presents the physics of portal imaging. The effects of photon energies, dose, noise, imaging geometry, and other factors on image quality are discussed. A basic understanding of these relationships is important in maintaining optimal operation of EPIDs. In Sec. III, the technologies employed in commercially available EPIDs are described. Section IV describes the pertinent steps that the medical physicist needs to take to commission an EPID for clinical imaging and to maintain it in optimal operating condition. In Sec. V, complicated issues related to clinical implementation and use are discussed. Several modes of clinical operation to achieve various degrees of improvement in setup accuracy are introduced; each with different requirements of manpower, software tools, and computer resources. In Sec. VI, basic cost analysis for clinical adaptation of the EPIDs is discussed. The section ends with a projection of future trends in the use of EPIDs. Section VII offers a summary and recommendations from the Task Group. 


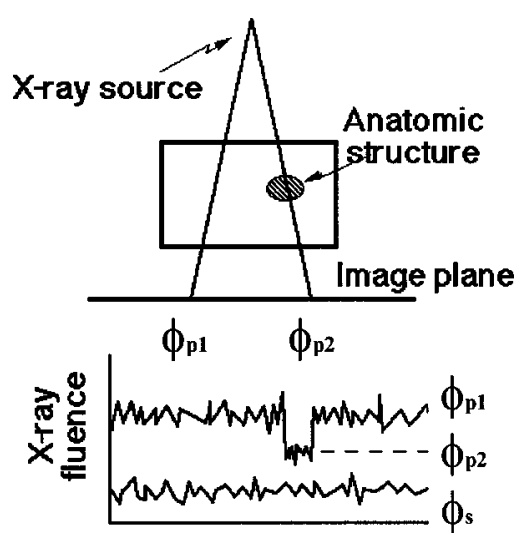

FIG. 1. Schematic representation of the imaging process. Fluences $\phi$ are defined in text.

\section{THE PHYSICS OF PORTAL IMAGING}

Treatment verification usually involves comparison of a portal image acquired during a treatment fraction with a reference image that is generated prior to the initiation of the treatment course. Sometimes, the first approved portal image is also used as the reference image. While the portal image is formed by the megavoltage beam used to treat the patient, the reference image can be kilovoltage (e.g., simulation film), megavoltage, or a digitally reconstructed radiograph.

It is generally accepted that the quality of images acquired using megavoltage $\mathrm{x}$ rays is inherently poorer than that acquired with kilovoltage $\mathrm{x}$ rays. Besides the well-known decrease in subject contrast (e.g., the differential attenuation between bone or air and soft tissues) as the energy of an $\mathrm{x}$-ray beam increases, many other factors contribute to the poor quality of portal images. These include the performance of the image receptor, $\mathrm{x}$-ray scatter due to patient thickness, the size of the x-ray source, noise in the human eye-brain system, and (indirectly) the position of the image receptor. The purpose of this section is to explain how these factors influence the portal image quality and to understand the fundamental limitations of imaging with megavoltage $\mathrm{x}$-ray beams. This in turn should help readers understand what they can and cannot expect from the imaging performance of EPIDs.

A number of key quantities give an objective measure of image quality. Figure 1 illustrates the image formation process and its relation to some key indicators of image quality. This section addresses contrast, noise, spatial resolution, detective quantum efficiency (DQE) of EPIDs, and x-ray scatter.

\section{A. Contrast}

Contrast, $C$, describes how much an object stands out from its surroundings and is defined as ${ }^{30}$

$$
C=\frac{\text { signal }}{\text { mean signal }}=\frac{\phi_{p_{2}}-\phi_{p_{1}}}{\left(\phi_{p_{2}}+\phi_{p_{1}}+2 \phi_{s}\right) / 2},
$$

where $\phi_{p_{1}}, \phi_{p_{2}}$, and $\phi_{s}$ are the primary and scatter photon fluences reaching the image receptor (Fig. 1). Motz and Danos have shown that this expression can be rewritten as ${ }^{30}$

$$
C=\frac{2\left(1-e^{-\Delta}\right)}{1+e^{-\Delta}+\frac{2 \mathrm{SF}}{1-\mathrm{SF}}},
$$

where $\Delta$ is the difference in attenuation between the object and the background (i.e., $\Delta=L_{x}\left|\mu_{\text {bone }}-\mu_{\text {water }}\right|$ ), $\mu_{\text {bone }}$ and $\mu_{\text {water }}$ are the x-ray attenuation coefficients for bone and water, respectively, $L_{x}$ is the thickness of the anatomic structure, and $\mathrm{SF}$ is the scatter fraction $\left\{\mathrm{SF}=\phi_{s}\left(\phi_{s}+\phi_{p}\right)\right\}$. Equation (1) shows that the contrast is increased by increasing the difference in attenuation along the x-ray path and is decreased by the addition of a scatter fluence.

Subject contrast of 1-cm-thick bone or air objects embedded within $20 \mathrm{~cm}$ of water as a function of x-ray energy can be calculated using Eq. (2). For simplicity, the contrast has been calculated assuming that no x-ray scatter occurs (i.e., $\mathrm{SF}=0$ ). For comparison purposes, $50 \mathrm{keV}$ approximates the mean energy of the $\mathrm{x}$-ray energy spectra used to generate a simulator image (100 kVp, diagnostic energy) and $2 \mathrm{MeV}$ that of the $6 \mathrm{MV}$ beam to generate a portal image. Examining the subject contrast at these two x-ray energies shows the subject contrast decreases from 0.5 to 0.037 (a factor of 13) for the bone and from 0.2 to 0.05 for the air pocket (only a factor of 4). This explains the enhanced visibility of the air passages relative to bony anatomy seen in the therapy image as compared to the simulator image.

Contrast is the result of differences in $\mathrm{x}$-ray attenuation within the patient. At low energies, the photoelectric process dominates. Since the photoelectric cross section is proportional to the atomic number raised to the third power $\left(Z^{3}\right)$, the higher atomic number of bone results in a larger attenuation coefficient compared to that of water. However, the photoelectric cross section is also inversely proportional to the energy cubed $\left(1 / E^{3}\right)$. Compton scattering becomes the dominant interaction process above $20 \mathrm{keV}$ for soft tissues and above $50 \mathrm{keV}$ for bone (assuming that the atomic number of bone is $\sim 13$ ). The Compton scattering cross section is dependent on the electron density of a material, which, except for hydrogen, varies only slightly with atomic number. The electron density of water $\left[\rho_{e}(\right.$ water $)=3.34$ $\times 10^{23}$ electrons $\left./ \mathrm{cm}^{3}\right]$ is comparable to that of bone $\left[\rho_{e}(\right.$ bone $)=5.81 \times 10^{23}$ electrons $\left./ \mathrm{cm}^{3}\right]$. Therefore, the difference in attenuation, and hence the contrast, reduces significantly at megavoltage energies.

\section{B. Signal-to-noise ratio}

\section{Quantum noise}

The most important concept to understand is that image quality (or "detectability" of bony anatomy) is ultimately determined not by the subject contrast of the object being imaged but by the signal-to-noise ratio (SNR) of the image. A number of sources of noise contribute to the SNR. A limiting source of noise is due to x-ray quantum statistics. This is best explained again with Fig. 1, which shows the process of $\mathrm{x}$-ray image formation. The difference in attenuation be- 
tween an object and its surroundings (i.e., subject contrast) results in different number of $\mathrm{x}$-ray quanta reaching and interacting in an image receptor. The subject contrast is determined by the energy of the $\mathrm{x}$-ray beam, the radiological properties of the object being imaged, and the amount of $\mathrm{x}$-ray scatter reaching the image receptor. However, since image formation is a statistical process involving the detection of discrete $\mathrm{x}$-ray quanta, there will be a statistical uncertainty (known as x-ray quantum mottle) in the number of $\mathrm{x}$-ray quanta that interact in the image receptor. The detectability of the object therefore depends not only on how large the difference in attenuation is between the object and its surroundings, but also on how large this signal difference is compared to the uncertainty in the signal, i.e., SNR.

The number of $\mathrm{x}$-ray quanta detected in some time interval follows Poisson counting statistics. For a Poisson process, the variance in the number of detected x-ray quanta is equal to the mean number of detected photons. Therefore, if the mean fluences are known, a signal-to-noise ratio can be calculated. The signal-to-noise ratio of the bone signal shown in Fig. 1 is calculated as

$$
\mathrm{SNR}=\frac{\text { image signal }}{\text { noise }}=\frac{\phi_{p_{2}}-\phi_{p_{1}}}{\sqrt{\left(\phi_{p_{2}}+\phi_{p_{1}}+2 \phi_{s}\right) / 2}} .
$$

Rewriting in terms of the geometry shown in Fig. 1, we obtain

$$
\mathrm{SNR}=\sqrt{A \phi_{i} T \eta} \frac{2\left(1-e^{-\Delta}\right)}{\sqrt{1+e^{-\Delta}+\frac{2 \mathrm{SF}}{1-\mathrm{SF}}}},
$$

where $A$ is the area of the detector element, $\phi_{i}$ is the incident fluence, $T$ is the patient transmission, and $\eta$ is the $\mathrm{x}$-ray detector efficiency. Equation (4) shows that the SNR, like the contrast, decreases as the difference in attenuation between the object and the background $(\Delta)$ decreases. However, unlike the contrast, the SNR is proportional to the number of $\mathrm{x}$ rays detected $\left(A \phi_{i} T \eta=\right.$ the area $\times$ fluence $\times$ transmission $\times$ collection efficiency $=$ number of detectedx rays). In addition, scatter reduces the SNR by adding noise without contributing to the signal.

The SNR versus $\mathrm{x}$-ray energy for an image of a $1-\mathrm{cm}$ thick bone in $20 \mathrm{~cm}$ of tissue can be calculated using Eq. (4). A typical diagnostic imaging procedure delivers a dose of $0.05 \mathrm{cGy}(50 \mathrm{mR})$ to the patient. ${ }^{31}$ For the same patient dose at megavoltage energies, the SNR would be $\sim 100$ times smaller. While the diagnostic SNR would satisfy Rose's criteria for visibility $(\mathrm{SNR}=5),{ }^{30}$ the megavoltage beam would not (Table I). However for the same photon fluence, a megavoltage beam delivers more dose. Doses more common in megavoltage imaging are also shown in Table I.

This simple model demonstrates that subject contrast decreases with increasing $\mathrm{x}$-ray energy. Not only does the contrast of objects decrease, the rate of decrease depends on the effective atomic number of the object. This results in the contrast of air passages exceeding that of bony anatomy
TABLE I. Calculated SNR and patient doses at diagnostic and therapeutic $\mathrm{X}$-ray energies.

\begin{tabular}{llllll}
\hline \hline Energy & $\begin{array}{c}\text { Diagnostic } \\
(50 \mathrm{keV})\end{array}$ & $\begin{array}{c}\text { Therapeutic } \\
(2 \mathrm{MeV})\end{array}$ & $\begin{array}{c}\text { Therapeutic } \\
(2 \mathrm{MeV})\end{array}$ & $\begin{array}{c}\text { Therapeutic } \\
(2 \mathrm{MeV})\end{array}$ & $\begin{array}{c}\text { Therapeutic } \\
(2 \mathrm{MeV})\end{array}$ \\
\hline $\begin{array}{l}\text { Patient } \\
\text { dose }\end{array}$ & $0.05 \mathrm{cGy}$ & $0.05 \mathrm{cGy}$ & $1 \mathrm{cGy}$ & $10 \mathrm{cGy}$ & $55 \mathrm{cGy}$ \\
SNR & 71 & $<1$ & 4.8 & 15 & 35 \\
\hline \hline
\end{tabular}

when x-ray energy exceeds $100 \mathrm{keV}$. Furthermore, the SNR of the bone signal decreases rapidly with increasing energy. For the same dose to the patient, the SNR is much lower at megavoltage energies $(2 \mathrm{MeV})$ than that at diagnostic energies $(50 \mathrm{keV})$. For typical diagnostic and therapy doses of 0.05 and $10 \mathrm{cGy}$, respectively, the gap in SNRs is reduced. The SNR is only five times lower at megavoltage energies.

\section{Quantum efficiency}

While quantum noise affects image quality, the efficiency of propagating the quanta through to the final detection stage can have a large impact on the SNR. An analysis of the detective quantum efficiency (DQE) of an imaging system determines the magnitude of this effect. While a thorough introduction in DQE is beyond the scope of this report (see, e.g., Ref. 32), a brief example of the impact of DQE on the design of one component of the imaging chain is presented. The DQE is a measure of how efficient the imaging system is at transferring the information contained in the radiation beam incident upon the detector. This is expressed as the square of the ratio of SNR output to SNR input as a function of spatial frequency.

The image receptor should always have high quantum efficiency so that a large fraction of the incident $\mathrm{x}$-ray quanta actually will interact in the receptor. In reality, portal imaging generally operates with low quantum efficiency. All commercial portal imaging systems use a metal plate (x-ray converter) to convert photons to Compton electrons. In video-based EPIDs, a phosphor screen is used to convert the electrons into optical photons. A scanning liquid ion chamber directly detects ionization due to the electrons. While $\sim 4 \%$ of the incident $x$-ray quanta interact in the metal plate, less than $1 \%$ of the incident $\mathrm{x}$-ray quanta will generate electrons that exit from the metal plate, propagating quanta further down the imaging chain. Figure 2 shows the quantum efficiency of a $1 \mathrm{~mm}$ copper plate in contact with different thickness of phosphor screens, when irradiated by a $2 \mathrm{MeV}$ x-ray beam (calculated using the EGS4 Monte Carlo code). Conventional portal film, exposed under a metal plate, with no phosphor, has a quantum efficiency of $\sim 1 \%$.

Figure 2 shows that the quantum efficiency increases as the thickness of the phosphor screen increases, because the incident $\mathrm{x}$-ray quanta can also interact directly within the phosphor screen. ${ }^{33}$ Therefore, somewhat fortuitously, the need for a phosphor screen increases the quantum efficiency of commercial EPIDs. For example, a phosphor screen thickness of $200 \mathrm{mg} / \mathrm{cm}^{2}$ (in a camera-based EPID) has a quantum efficiency $\sim 2.5$ times greater than the conventional cassettes 


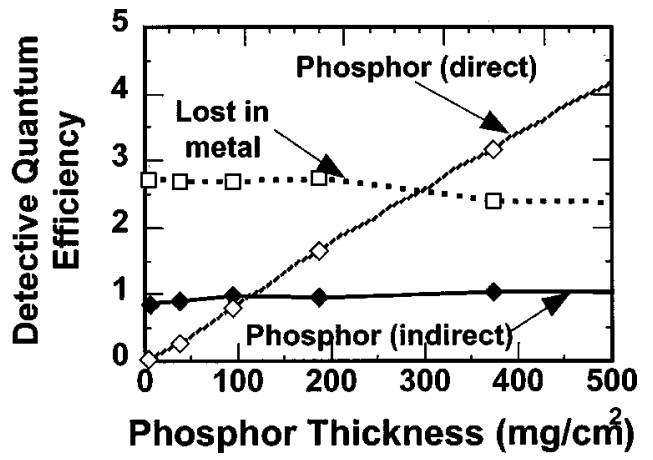

FIG. 2. The percentage of incident x-ray quanta that deposit energy in the phosphor layer of an x-ray detector consisting of a $1 \mathrm{~mm}$ copper plate and different thicknesses of $\mathrm{Gd}_{2} \mathrm{O}_{2} \mathrm{~S}$ phosphor screens. The "phosphor (indirect)' " curve represents those quanta that first interact in the copper plate and deposit energy in the phosphor screen. The "lost in metal" curve represents those quanta that interact in the metal plate but do not deposit energy in the phosphor screen. These quanta do not contribute to the image. As the phosphor thickness increases the number of x-ray quanta that deposit energy directly in the phosphor layer also increases.

used for portal films. A similar argument can be made for the liquid in the scanning ion chamber systems, with a thickness of $\sim 80 \mathrm{mg} / \mathrm{cm}^{2}$, yielding a quantum efficiency of 1.5 relative to film.

Direct approaches to increase quantum efficiency by increasing the thickness and/or density of the metal plate x-ray detectors are often ineffective. Typically, spatial resolution deteriorates due to the increased extent of the x-ray deposition region. For the commercial camera-based EPIDs, thick phosphor screens are often employed. In addition to the loss of spatial resolution and optical light transmission, thick screens are prone to nonuniformity in phosphor content, and thus add to the structure noise of the imaging system. It is unlikely that increasing the thickness of the phosphor screens will yield further benefits.

\section{Other sources of noise}

The above-given analysis of SNR and quantum efficiency is based on primary $x$-ray quantum noise only and does not include other sources of noise, each of which can have a major effect on the image quality. There are a large number of other noise sources in any portal imaging system, including energy absorption noise, ${ }^{34}$ noise added by the imaging system, and noise in the human visual system.

Note that the small amount of information from the x-ray beam extracted by all EPIDs and portal films still represents a very large amount of detected x-ray quanta. Indeed, at typical exposure (or dose) used for imaging, the x-ray fluence reaching the image receptor is generally 100 times greater at megavoltage energies than at kilovoltage energies. ${ }^{35}$ It appears that poor image quality is not because the image receptors do not have enough x-ray quanta interacting in them, but because the image receptors either add additional noise to the images or display the images so that noise in the eye-brain system becomes important.

Measurements of Munro et al. ${ }^{36-38}$ suggest that conventional portal films record more information than EPIDs, but a)

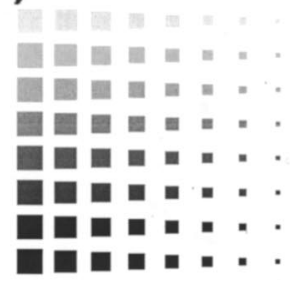

b)

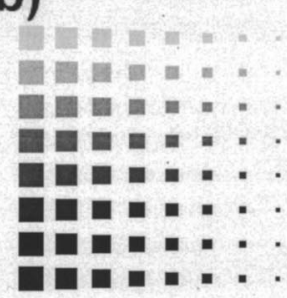

c)

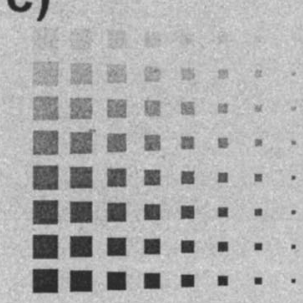

e) d)

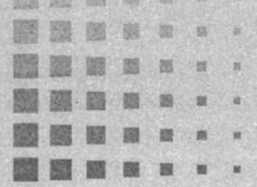

f)
FIG. 3. Images calculated for various stages of the image acquisition process: (a) represents the input image, (b) the image that would be generated using all the incident x-ray quanta, (c) the image that would be generated using the x-ray quanta detected by the image receptor, (d) the image that would be generated after accounting for energy absorption noise, and (e) and (f) the addition of noise by the image receptor. The additive noise increased the variance in (e) by a factor of 4 and that in (f) by a factor of 9 .

the experience of EPID users and contrast-detail studies ${ }^{39}$ suggest that improved display of portal images by EPIDs reduces the effect of observer noise ${ }^{40}$ inherent in visual film observation. This is due to the superior contrast resolution of the EPID and the ability to process the images and more than compensates for the smaller information content.

The ideal image receptor would be an EPID or film that adds no electronic or film noise to the image and which displays the image optimally. Recent developments, such as EC-L film and amorphous silicon EPIDs, come close to meeting this ideal. Figures 3(a)-3(f) show a series of simulated images to demonstrate visually the effect that noise can have on the appearance of x-ray images. Figure 3(b) shows the ideal detector where the noise of the image of the objects in Fig. 3(a) is due to the statistical variation of the x-ray quanta. The change in image quality from Fig. 3(c) to Fig. 3(d) shows the effect of energy absorption noise. The change in image quality from Fig. 3(d) to Fig. 3(e) shows the effect of noise added by the imaging system. The further change in image quality in Fig. 3(f) shows the effect of noise from the human visual system. The amount of noise added by each stage of the imaging system is representative of a typical video-based EPID. 


\section{Spatial resolution}

Another important factor that influences image quality, but which is not included in the above-described model, is spatial resolution. Spatial resolution is a measure of how the image signal is blurred by the imaging system. For example, the spatial resolution of the system influences how well edges, such as those resulting from bones, will be detected. The spatial resolution of commercial EPIDs depends on factors that are common to all EPIDs as well as factors that are device specific. The spread of high energy particles in the metal plate is common to all commercial EPIDs and is quite modest. ${ }^{41,42}$ In addition to the lateral migration of high energy electrons, other processes such as x-ray scatter, bremsstrahlung, and positron annihilation, also contribute to the signal spread in the metal plate. ${ }^{33,36,42}$ Once the high energy particles exit from the metal plate they can spread in the convertor (phosphor screen, ionizing fluid). While lateral electron migration would be greater in the ionizing fluid $\left(\sim 0.8 \mathrm{~g} / \mathrm{cm}^{3}\right)$ than in the phosphor screen $\left(\sim 3.74 \mathrm{~g} / \mathrm{cm}^{3}\right)$, it is light spread in the phosphor screen ${ }^{36}$ that mostly determines the spatial resolution for the camera-based EPIDs. Pixel size is the primary factor that determines the spatial resolution for the matrix ion chamber EPID. ${ }^{43}$

The spatial resolution of an imaging system is often characterized by examining how well the system reproduces a point object (infinitesimally small). Acquiring an image of such a point object measures the system's point spread function. Conventionally, this spread of signal is represented in the form of the modulation transfer function (MTF). The MTF describes how well the system passes different spatial frequencies and is calculated from the Fourier transform of the point spread function. Any complete characterization of an imaging system requires an examination of both the signal-to-noise characteristics and the spatial frequency response of the system.

It is a common misconception that the spatial resolution of the imaging system is the major factor limiting the image quality of portal films and portal images. Spatial resolution of any portal image depends upon three quantities, the size of the $\mathrm{x}$-ray source, the spatial resolution of the image receptor, and image magnification. Source sizes of medical linear accelerators have been measured to be $\sim 1 \mathrm{~mm}$ full width at half maximum, or smaller. Other measurements have shown that the line-spread functions for camera-based EPIDs are $0.8-1.0 \mathrm{~mm}^{36,44}$ full width at half maximum while that for the matrix ion chamber EPID is $1.5-2.0 \mathrm{~mm} .{ }^{43}$ Image magnification is variable and can have an important effect on the spatial resolution of the system. As the magnification increases, geometric blurring due to the x-ray source increases, while the size of the patient anatomy projected at the plane of the image receptor also increases, reducing the effect of blurring by the image receptor. Thus, there is an optimal image magnification where the blurring due to both the image receptor and the $\mathrm{x}$-ray source is minimized. Calculations suggest that the optimal image magnification is between 1.3 and 2.0, which fortunately encompasses the range of operation for almost all commercial EPIDs. ${ }^{4,46}$
Finally, in portal imaging, it is important to recognize that there is reduced attenuation at megavoltage energy (compared with kilovoltage), which results in the reduced sharpness of the object and an apparent change in the projected object dimension. This leads to the perception that portal images have lower spatial resolution than diagnostic images. Care must be taken when comparing images acquired with different photon energies.

\section{X-ray scatter}

Scattered x rays, or any "nonprimary" photons, can reduce the subject contrast and the signal-to-noise ratio of portal images (see Fig. 1) by generating signals in the image receptor that carry no geometric information about the patient's anatomy but that add noise to the images. The reduction of contrast by $\mathrm{x}$-ray scatter is of serious concern for portal films, since the display contrast of film cannot be adjusted to compensate for any reduction in subject contrast. For EPIDs, the reduction in signal-to-noise ratio due to $\mathrm{x}$-ray scatter is more important than the reduction in contrast. While x-ray scatter has long been a major concern in kilovoltage $\mathrm{x}$-ray imaging, it has been shown that it is much less of a problem for megavoltage portal imaging. ${ }^{45,47}$ As the energy of the x-ray beam increases, the scatter fraction (the fraction of the total fluence reaching the image receptor that is due to scattered $\mathrm{x}$ rays) decreases from 0.9 at $100 \mathrm{keV}$ to less than 0.6 at $6 \mathrm{MV}$ (at the exit surface of the patient). (On the other hand, the scattered component of kilovoltage beams can be reduced substantially using grids, which is not possible for megavoltage beams.) As in diagnostic radiology, geometric factors are quite important in influencing the scatter fluence reaching the image receptor at megavoltage energies. The scatter fraction increases as the patient thickness increases, as the field size increases, and as the air gap between the patient and the image receptor decreases. Apart from extreme situations such as very large patient thicknesses and field sizes, and small air gaps, x-ray scatter generally does not degrade the image quality of portal image significantly. Jaffray et al. have shown, using Monte Carlo calculations, that the signal-to-noise ratio would improve by less than $10 \%$ if all $\mathrm{x}$-ray scatter were eliminated before reaching the image receptor when a moderately thick (20 $\mathrm{cm})$ patient is irradiated. ${ }^{47}$

\section{THE TECHNOLOGY OF MEGAVOLTAGE IMAGING}

Many different EPIDs have been examined since the early 1980 s as alternatives to film for megavoltage imaging. Readers are referred to four comprehensive reviews of portal imaging devices for further details. ${ }^{48-51}$ The following discussion on EPIDs will concentrate on features of the matrix ion chamber and the camera-based EPIDs, which are both available commercially. Promising new systems based on active matrix flat panel imaging (AMFPI) technology will become available commercially in the near future and are detailed in the literature. ${ }^{52-61}$ The new AMFPI systems will perform as well as or better than the EPID systems described here. 


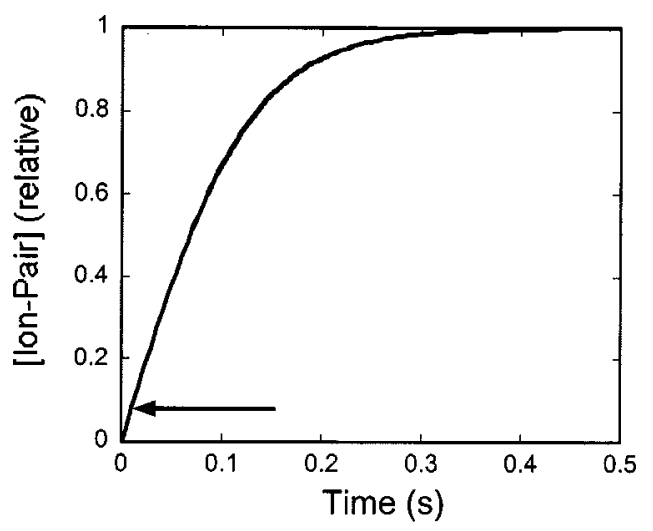

FIG. 4. The relative ion-pair concentration in the matrix ion chamber as a function of irradiation time - in the absence of high voltage. The ion-pair concentration builds up to a maximum value in $0.3-0.5 \mathrm{~s}$ and does not change with increasing irradiation time. The equilibrium concentration depends on dose rate. The horizontal arrow represents the signal that would be measured in a $10 \mathrm{~ms}$ period (the typical time that high voltage is applied to a high voltage electrode) if no charge integration occurred in the ionizing fluid.

\section{A. Matrix ion chamber}

The matrix ion chamber device (originally developed by Meertens, van Herk and their colleagues) consists of two sets of electrodes that are oriented perpendicularly to each other separated by a $0.8 \mathrm{~mm}$ gap, which is filled with a fluid (2,2,4-trimethylpentane) that is ionized when the device is irradiated. ${ }^{62}$ Each set of electrodes consists of 256 wires spaced $1.27 \mathrm{~mm}$ apart to provide an active area of $32.5 \mathrm{~cm}$ on a side. One set of electrodes is connected to 256 electrometers and the other set of electrodes is connected to a highvoltage supply that can apply a $300 \mathrm{~V}$ potential to each electrode individually. The matrix ion chamber array is read out by applying a high voltage to each of the high-voltage electrodes in succession (for approximately $20 \mathrm{~ms}$ ) and measuring the signal generated in each of the 256 signal electrodes. This procedure takes $5.5 \mathrm{~s}$ to read out an image. In addition, a fast (lower resolution) scanning mode is available that scans the array in $1.5 \mathrm{~s}$ by applying the high voltage for a 10 ms period to two high voltage electrodes at a time. The fast acquisition mode is useful for acquiring double-exposure images. The more recent systems operates with a high voltage bias of $500 \mathrm{~V}$ and at rate of $5 \mathrm{~ms}$ readout per electrode giving an entire image read out time of $1.25 \mathrm{~s}$.

The most obvious advantage of the matrix ion chamber is its compact size, which makes the device a convenient replacement for film cassettes. Another advantage is its geometric reliability-images acquired with the system have no geometric distortions. The major limitation of a scanning radiation detector is quantum utilization, since only one highvoltage electrode (out of 256) is active at any one time. However, the physics of signal generation in the 2,2,4trimethylpentane improves the quantum utilization of the matrix ion chamber considerably. The signal measured by the matrix ion chamber depends on the rate of formation and the rate of recombination of the ion pairs that are generated in the ionizing fluid. Even when no high voltage is applied to

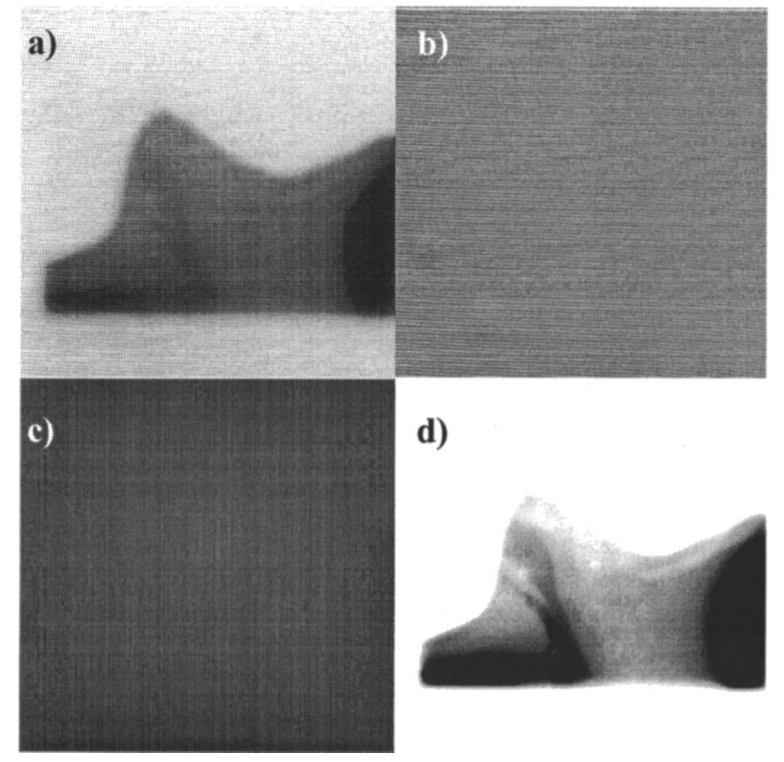

FIG. 5. (a) Raw image from a matrix ion chamber EPID. (b) Dark field image from this EPID. The signals are mostly caused by transients of the high voltage switching. (c) Flood field image from this EPID. The vertical lines are due to differences in sensitivity of the amplifiers. (d) Fully corrected image (single frame at 1.7 s $4 \mathrm{MV} 250 \mathrm{MU} / \mathrm{min}$ ).

the electrodes, the rate of recombination of the ion pairs generated in the 2, 2, 4 trimethylpentane is relatively slow. Therefore, the concentration of ion pairs can increase over a period of time until an equilibrium is reached between ionpair formation, and is a function of the dose rate at the matrix ion chamber and ion-pair recombination, the latter is proportional to the square of the ion-pair concentration. The rate of ion-pair formation as a function of irradiation time in the absence of high voltage bias is shown in Fig. 4.

In effect, the signal measured by any electrode of the matrix ion chamber does not depend greatly on the dose rate during the 5-20 ms period when the high voltage is applied but on the previous irradiation history of the electrode. However, the effective period of the charge integration $(0.5 \mathrm{~s})$ is still short compared with the total image acquisition time. Therefore, a large fraction of the radiation that interacts with the matrix ion chamber does not generate any measurable signal. For this reason, the matrix ion chamber requires higher doses to generate images than other portal imaging devices. Note that once the latent image has been formed, the more rapidly that the image can be read out, the smaller the dose to the patient required to form an image.

An example of a lateral neck image acquired with the matrix ion chamber EPID is shown in Fig. 5. Since spurious (dark) signals can be generated in the electrometers and ion chambers, and because the sensitivities of each ion chamber can vary, the raw signals from the matrix ion chamber EPID must be processed before yielding a usable image. For similar reasons, calibration of the system on a monthly basis ensures its optimal operation. Because the matrix ion chamber is a scanning EPID, it is susceptible to artifacts if the dose rate of the accelerator changes during image acquisition. Thus, the radiation beam has to stabilize for some pe- 
(a)

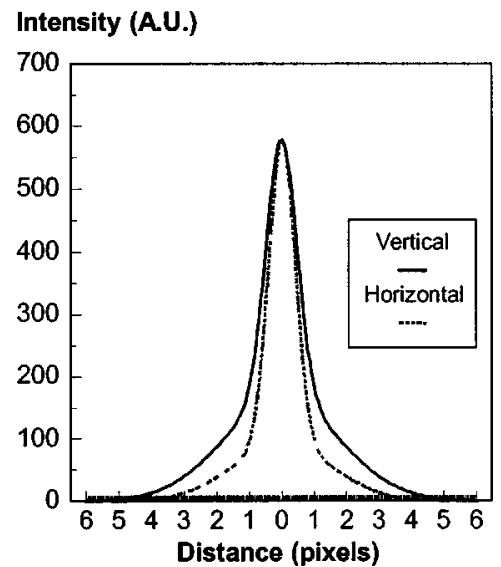

(b)

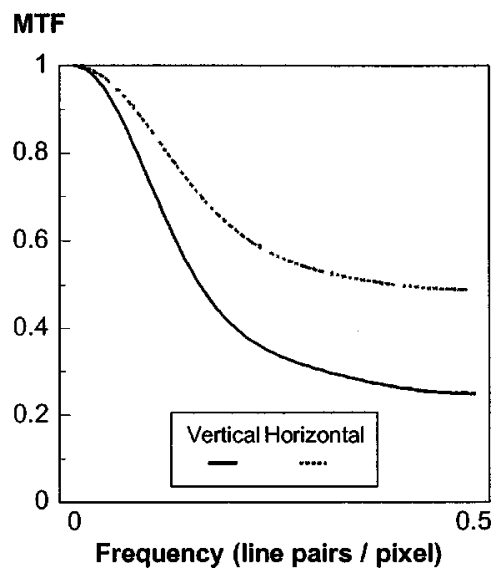

FIG. 6. (a) Fitted presampling line spread functions (LSF), normalized on the central value. (b) Modulation transfer functions (MTF). The poorer vertical resolution may be caused by the relatively low speed of the electrometer amplifiers and the shape of the electric field in the chamber. riod (typically $1.0 \mathrm{~s}$ ) after start-up before image acquisition can begin. The best image quality results when the scanning of the high-voltage electrodes is synchronized with the pulsing of the linear accelerator. In practice, the matrix ion chamber EPID needs to be calibrated for each of the dose rates of the accelerator that will be used clinically. Finally, many of the radiation sensitive readout electronics are located immediately adjacent to the active region of the matrix ion chamber. Even with the use of electronic components that have improved resistance to radiation damage, care must be used to ensure that the field size or the position of the EPID is coordinated to prevent accidental irradiation of the electronics. $^{63}$

Van Herk et al. have characterized the MTF and DQE of the system by correcting for the nonlinear response of the system. Figures 6(a) and 6(b) show the fitted presample line spread function and the corresponding MTF of the latest matrix ion chamber EPID. Horizontal and vertical directions are with respect to the image detector. The detector has a high sensitivity all the way up to the Nyquist frequency. Two effects may cause the significant difference between the horizontal and vertical resolution. First, the 256-electrometer amplifiers include a filter with a time constant of about $1 \mathrm{~ms}$, which may cause some blurring in the vertical direction. Second, the absence of shielding between the ionization chambers may cause some spurious sensitivity outside the pixel area due to the direction of the electric field lines.

The zero-frequency DQE depends strongly on the dose per image (Fig. 7). In contrast to linear detectors, where the

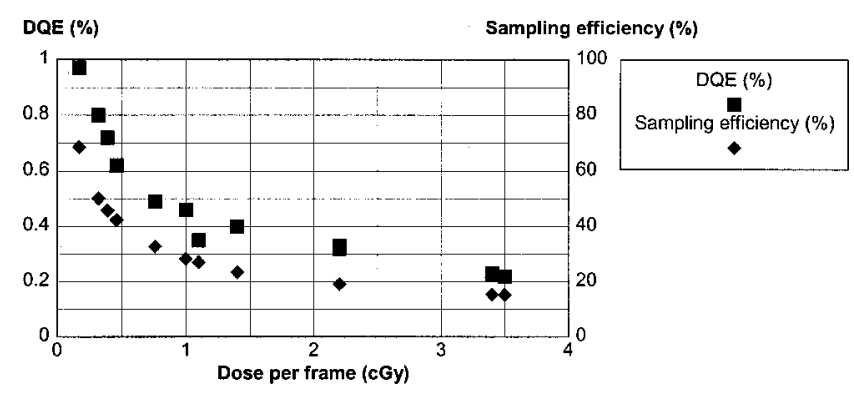

FIG. 7. Detective quantum efficiency (DQE) and sampling efficiency of the matrix ionization chamber device.
DQE decreases with decreasing dose (due to the influence of system noise), the DQE increases for this detector. This effect is caused by the increase in integration time at lower dose rates due to the latent image in the liquid. The ratio between the DQE and the sampling efficiency gives the inherent efficiency of the metal plate detector, which would be reached at $100 \%$ sampling efficiency. Decreasing the readout time (which improves the sampling efficiency) may therefore further improve the DQE. Efforts are being made to further characterize the frequency dependence of the DQE for the matrix ion chamber EPID.

In addition to the detection electronics, a typical liquid ion chamber EPID has a gantry mounted robotic arm that provides complete retraction of the unit.

\section{B. Camera-based EPIDs}

Camera-based systems consist of a metal plate and a phosphor [gadolinium oxysulfide $\left(\mathrm{Gd}_{2} \mathrm{O}_{2} \mathrm{~S}\right)$ ] screen viewed by a camera using a $45^{\circ}$ mirror. When irradiated, highenergy electrons generated in the metal plate and the phosphor screen are converted into light in the phosphor screen and this light creates the video signal generated by the camera. The video signal from the camera can be digitized and the digitized image can be viewed on a monitor located in the control area of the accelerator. The video systems differ primarily in the deployment of their housing assembly (see Table II) and camera operation. Various techniques for readout are designed to reduce the impact of noise in the imaging chain.

Video EPIDs suffer from the major limitation of light collection efficiency of the optical chain. Since the light is highly scattered within the phosphor screen, the light is emitted from the rear of the screen in all directions with equal probability. Only those light photons that are emitted within a small cone subtended by the lens of the camera can generate a signal in the camera; typically only $0.1 \%-0.01 \%$ of the light emitted by the phosphor screen reaches the camera. This poor light collection efficiency reduces image quality in two ways. First, if an x-ray photon interacts in the x-ray detector but none of the light generated by this interaction reaches the camera, then no measurable signal is produced. 
TABLE II. Features of the five commercially available EPIDs.

\begin{tabular}{|c|c|c|c|c|c|}
\hline Supplier & Elekta-Philips & Eliav & Infimed $^{\mathrm{a}}$ & Siemens & Varian \\
\hline Name & SRI 100 & PortPro & Theraview & Beamview Plus & PortalVision \\
\hline Type & CCD camera & CCD camera & Plumbicon camera & Newvicon camera & Matrix ion chamber \\
\hline Detector pixels & $512 \times 512$ & $512 \times 512$ & $512 \times 512$ & $512 \times 512$ & $256 \times 256$ \\
\hline Digitization & 8 bit frame-grabber & 8 bit frame-grabber & 8 bit frame-grabber & 8 bit frame-grabber & 14 bit $\mathrm{A} / \mathrm{D}$ converter \\
\hline $\begin{array}{l}\text { Max frequency } \\
\text { of acquisition }\end{array}$ & 7 frames/s & 30 frames/s & 2 monitor units & 30 frames/s & $\begin{array}{l}\text { Mark 1: } 5.5 \mathrm{~s} \\
\text { Mark 2: } 1.25 \mathrm{~s}\end{array}$ \\
\hline $\mathrm{X}$-ray detector & $\begin{array}{l}1.5 \mathrm{~mm} \text { steel plate } \\
+411 \mathrm{mg} / \mathrm{cm}^{2} \\
\mathrm{Gd}_{2} \mathrm{O}_{2} \mathrm{~S} \text { screen }\end{array}$ & $\begin{array}{l}1.5 \mathrm{~mm} \text { steel plate } \\
+411 \mathrm{mg} / \mathrm{cm}^{2} \\
\mathrm{Gd}_{2} \mathrm{O}_{2} \mathrm{~S} \text { screen }\end{array}$ & $\begin{array}{l}1.5 \mathrm{~mm} \text { brass plate } \\
+400 \mathrm{mg} / \mathrm{cm}^{2} \\
\mathrm{Gd}_{2} \mathrm{O}_{2} \mathrm{~S} \text { screen }\end{array}$ & $\begin{array}{l}1.2 \mathrm{~mm} \text { brass plate } \\
+160 \mathrm{mg} / \mathrm{cm}^{2} \\
\mathrm{Gd}_{2} \mathrm{O}_{2} \mathrm{~S} \text { screen }\end{array}$ & $\begin{array}{l}1.0 \mathrm{~mm} \text { platoferrite plate } \\
+0.8 \mathrm{~mm} 2.24 \text {-trimethyl-pentane } \\
+ \text { wire electrodes }\end{array}$ \\
\hline $\begin{array}{r}\text { Mechanical } \\
\text { assembly }\end{array}$ & Dismountable & Portable & $\begin{array}{l}\text { Partly retractable and } \\
\text { partly dismountable }\end{array}$ & Fully retractable & $\begin{array}{l}\text { Fully retractable; portable } \\
\text { if used with retractable arm }\end{array}$ \\
\hline Mounting & Philips only & Any accelerator & $\begin{array}{l}\text { Any accelerator (GE, } \\
\text { Varian, Scanditronix) }\end{array}$ & Siemens only & Any accelerator (attached by customer) \\
\hline $\begin{array}{l}\text { Collision } \\
\text { interlock }\end{array}$ & Yes & No & $\begin{array}{l}\text { Yes (connect to } \\
\text { accelerator motion } \\
\text { interlocks) }\end{array}$ & $\begin{array}{l}\text { No (interlock } \\
\text { activated during } \\
\text { deployment only) }\end{array}$ & Yes \\
\hline $\begin{array}{l}\text { Field of view } \\
\text { at isocenter } \\
(\mathrm{cm} \times \mathrm{cm})\end{array}$ & $\begin{array}{l}\text { Fixed } \\
19 \times 24\end{array}$ & Variable & $\begin{array}{l}\text { Adjustable } \\
\text { 31.8 diam Varian } \\
\text { 31.5 diam Sanditronix } \\
\text { 31.6 diam G.E. }\end{array}$ & $\begin{array}{l}\text { Fixed } \\
24 \times 30\end{array}$ & $\begin{array}{l}\text { Adjustable } \\
25 \times 25\end{array}$ \\
\hline $\begin{array}{l}\text { Detector area } \\
\qquad(\mathrm{cm} \times \mathrm{cm})\end{array}$ & $30 \times 38$ & Variable & $40 \times 40$ (detector) & $35 \times 44$ (detector) & $32.5 \times 32.5$ (detector) \\
\hline $\begin{array}{l}\text { Detector to } \\
\quad \text { isocenter }(\mathrm{cm})\end{array}$ & 60 & Not applicable & $\begin{array}{l}\text { 30-60 (Varian) } \\
26-67 \text { (G.E.) } \\
27-78 \text { (Scanditronix) }\end{array}$ & 39 & $5-80$ \\
\hline $\begin{array}{l}\text { Display center } \\
\text { accuracy }\end{array}$ & $\pm 1 \mathrm{~mm}$ & & $\pm 5 \mathrm{~mm}$ & $\pm 2 \mathrm{~mm}$ & $\pm 5 \mathrm{~mm}$ \\
\hline $\begin{array}{l}\text { Prototype } \\
\text { descriptions }\end{array}$ & Ref. 49 & & Ref. 51 & Ref. 53 & Refs. 46,47 \\
\hline $\begin{array}{l}\text { Resolution } \\
\text { 1p/mm (Sec. IV) }\end{array}$ & 0.180 & 0.305 & 0.223 & 0.204 & 0.258 \\
\hline
\end{tabular}

${ }^{a}$ The Infimed system is now marketed by Cablon Medical.

Second, if only a small signal is produced in the camera, then noise generated by the preamplifier and other electronics of the camera may be large compared to the signal. As a result, the development of commercial camera-based EPIDs has focused on increasing light collection of the optical chain by increasing the thickness of the phosphor screen to increase the light output and to a smaller extent increase the x-ray quantum efficiency, ${ }^{39,44}$ and using a large aperture lens that collects more of the light. ${ }^{39,49}$

The use of large aperture lenses suffers from decreased spatial resolution because of spherical aberrations (light rays reaching the edges of the lens do not focus to the same point as those reaching the center). The spatial resolution of these lenses decreases from the center to the edge of the lens. There is also a reduced depth of field which renders the focal distance more sensitive to optical wavelength. Large aperture lenses also suffer from vignetting, which results in images that are brighter at the center of the lens than the edge. This change in image brightness is corrected through software or hardware schemes. Finally, large aperture lenses can generate distortions, such as pin cushion or barrel distortion, which cause straight lines to appear curved in the image, especially at the edges of the field of view. Examples of the MTF and zero frequency DQE of a camera-based EPID from camera-based system are shown in Fig. 8. An image acquired with two-monitor units at $6 \mathrm{MV}$ with this system is shown in

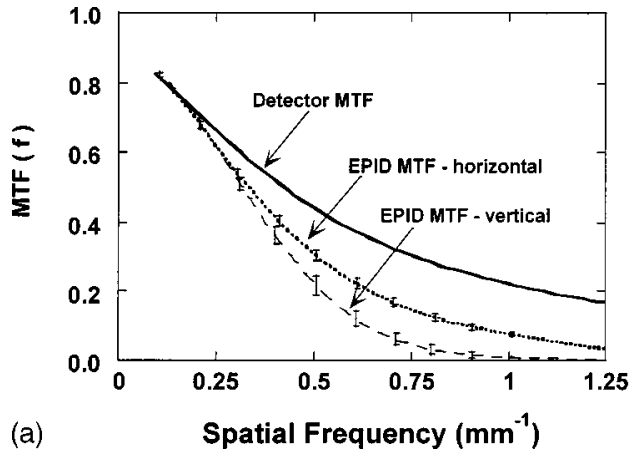

(a)

Medical Physics, Vol. 28, No. 5, May 2001

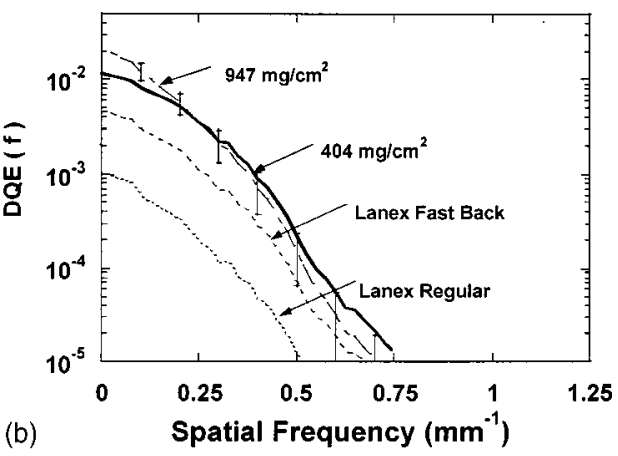

FIG. 8. (a) MTF, video EPID, (b) dQE, video EPID. 


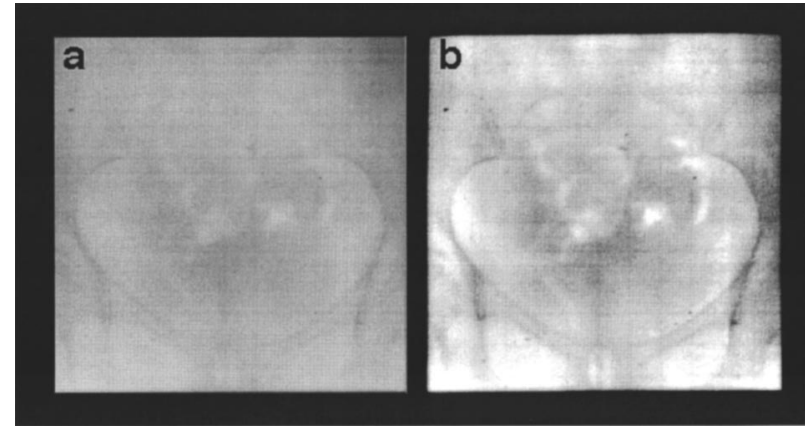

FIG. 9. Video EPID image (a) and (b) with enhancement.

Fig. 9. Image 9(a) was corrected for lens vignetting, while image 9(b) shows improvement from simple image enhancement tools such as level and window and contrast adjustment.

There are a variety of mounting systems for video-based EPIDs that range from rigid gantry mounts, partially or completely retractable systems to systems independent of the gantry on a portable stand.

\section{COMMISSIONING AND QUALITY ASSURANCE FOR EPIDs}

\section{A. Installation and commissioning}

At the time of installation/acceptance the following features must be verified: mechanical and electrical safety, geometric reproducibility, image quality, and software performance specifications. Following acceptance, commissioning will characterize operational features relevant to clinical use and specifications for routine quality assurance. The items discussed in detail here are summarized in Table III.

Some elementary safety aspects of EPID should always be checked, even if the devices are not used regularly. While one should adhere to the manufacturer's maintenance manual, if available, the following list contains a few of the basic tests that should be considered.

(a) Mechanical stability and integrity of EPID mounting and casing. The most serious risk is dropping the device on a patient or therapist during gantry rotation. Particular attention should be placed on checking the mounting point for detachable EPIDs and gears for retractable or movable EPIDs.

(b) Operation of collision detection system. The most serious potential hazard is the EPID colliding with the patient.

(c) Electrical insulation/grounding. The most serious potential hazard is potential electrocution of patient or staff. Most systems are grounded through the power outlet connected to the control computer and/or interface unit. The power supply insulation must be checked. One should also examine the cabling to the detector. The Varian PortalVision Mark 1 carries $300 \mathrm{~V}$ to a plug-on detector cassette (but the improved Mark 2 generates the applied $500 \mathrm{~V}$ internally from the $+15 \mathrm{~V}$ on the cable). Any moving cable or cables that potentially reach the patient or staff should be inspected visually once a month.

The Varian PortalVision detector contains a volatile liquid. In case of a collision, the device should be powered off and should be checked for any damage to the detector array. However, such damage is relatively unlikely since the actual array is under $2 \mathrm{~cm}$ of Styrofoam. Leakage of the liquid can be identified by a large change of the sensitivity of the central part of the detector. In such a case, the detector should be removed from service.

\section{Dose control}

Optimizing the dose necessary for imaging is important and varies by application and EPID. Improper dose control could cause failure to complete acquisition of a useful verification image in the preset dose (resulting in a useless image and extra dose required for obtaining a subsequent image), and over-dosage due to a failed beam-off signal. Most EPIDs

TABLE III. Summary of initial commissioning items, tolerances and methods.

\begin{tabular}{|c|c|c|c|}
\hline Item & Purpose & Tolerance & Method/tools \\
\hline Mechanical stability & $\begin{array}{l}\text { Safety } \\
\text { Image quality }\end{array}$ & $\begin{array}{l}\text { No accidental crash } \\
\text { Optical/physical alignment }(2 \mathrm{~mm})\end{array}$ & $\begin{array}{l}\text { Inspection } \\
\text { Optical test pattern } \\
\text { Optical distance indicators }\end{array}$ \\
\hline Electrical connections & Safety & No exposed connections/wires & Inspection of cabling/grounds \\
\hline Calibration & Image quality & Acceptable flat field, dark current/noise characteristics & $\begin{array}{l}\text { Per vendor follow calibration steps for energy, } \\
\text { field size, and noise. Vendor specifications vary. }\end{array}$ \\
\hline Dose control & Image quality/Safety & Preset dose (linac) control functions & $\begin{array}{l}\text { Program and verify correct beam termination } \\
\text { with dose. }\end{array}$ \\
\hline Image quality & Image quality & $\begin{array}{l}\text { Acceptable contrast }(1 \%) \\
\text { and spatial resolution }(2-3 \mathrm{~mm})\end{array}$ & $\begin{array}{l}\text { Las Vegas phantom, other contrast phantoms, } \\
\text { imaged at each energy }\end{array}$ \\
\hline Analysis software & Quantitative reporting & $\begin{array}{l}\text { Reported measurement within tolerance } \\
\text { of } 3 \mathrm{~mm} \text { and } 2 \mathrm{deg} \text {. Edge detection matches } \\
\text { field boundary }\end{array}$ & $\begin{array}{l}\text { Set up known error conditions and verify system } \\
\text { reporting and field edge definition under varying } \\
\text { field acquisition conditions }\end{array}$ \\
\hline
\end{tabular}


have adjustable trigger levels or delay times to allow the accelerator output to become stable. ${ }^{5,6}$ The dose delivered for a localization image can be preset in three ways: by manual beam interruption (not preferred, since operator errors might lead to a large dose), by a preset dose, or by auto-beam off. One should test correct image acquisition with different attenuators or an anthropomorphic phantom in the beam. Reducing the dose required for localization images is possible in video systems by using short exposure times (with some reduction in image quality), but the PortalVision has a predetermined acquisition time. For the latter, the use of a low dose rate is desirable. A complete test of the EPID-linear accelerator control system including the information system, which may contain parameters that are downloaded to the EPID or linac, must be performed prior to clinical use.

\section{Calibration}

Most EPID systems require some form of image calibration. Calibration provides correction factors and measures accelerator and EPID characteristics that are used to produce the highest quality image in routine use. Often, background signals are subtracted and inhomogeneity of response as well as linear accelerator beam characteristics are divided out. One should be aware that noise in the calibration images can reduce clinical image quality and should be minimized. The EPID must be calibrated for the varying conditions of clinical image acquisition. Calibration procedures depend on the type of EPID and vendor recommendations, however in each case it involves exposing the EPID to radiation under specific conditions. Calibration usually includes measurement of a dark current or noise image. This is acquired with no beam and represents signal present in the EPID when there is no radiation beam. This is followed by the acquisition of a full open field. The open field image is used to correct for reproducible treatment field specific characteristics, such as variation of intensity across a beam profile. Since beam characteristics may be beam energy and field size dependent, calibrations at various energies and field sizes must also be made. The information is used to generate correction factors used in the image acquisition process. In some cases, scatter and attenuation introduced by the patient can affect image quality and patient thickness and detector distance are therefore considered calibration parameters. The EPID may even require gantry angle calibration, if the mechanical stability of the EPID is such that a mechanical shift offsets the calibration of a flat field, or the treatment machine characteristics change significantly at varied gantry angles. The user is encouraged to determine which characteristics are most important for the EPID chosen, to ensure optimal operation.

Test image acquisition should be performed using the fresh calibration to ensure absence of artifacts due to accelerator instability or objects in the beam. While table grids and patient supporting plates appear as distractors in images, they are never sufficiently stable to be removed by calibration. The frequency of recalibration depends on the measured stability of image performance. Typically, a monthly recalibration may be necessary depending on the mechanical sta-

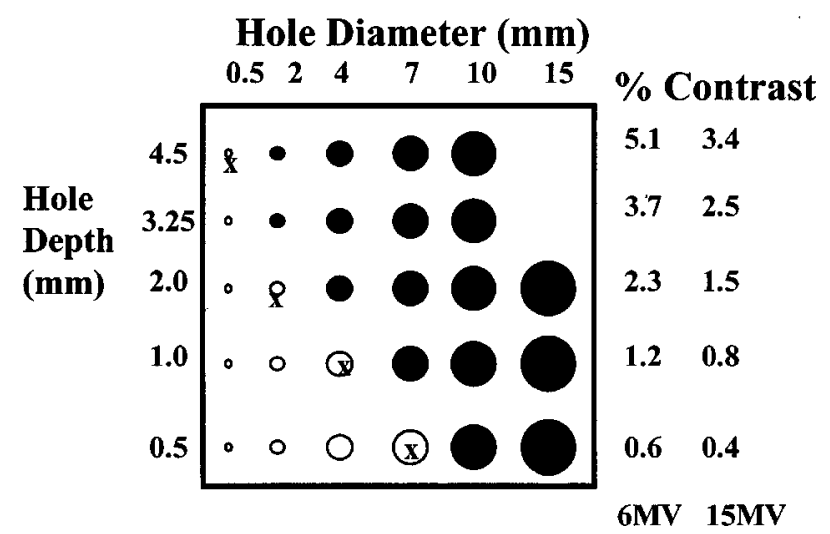

FIG. 10. Aluminum Las Vegas phantom for EPID image contrast and spatial resolution.

bility of the device. If any of the optical components in a fluoroscopic system are altered, a recalibration is recommended.

\section{Linearity}

The linearity of imaging geometry should be established during commissioning. Spatial distortions must be characterized or removed from EPID images before they can be used for quantitative portal imaging. Lack of rigidity in EPID components of video systems may result in instability of magnification or spatial linearity. EPID systems that use an analog video camera are susceptible to distortions due to variations in magnetic field and may depend on gantry angle. Bending or displacement of mirrors or front screens may also cause distortions. Simple mechanical phantoms (square grid of pins) to test for distortions are available from the manufacturer or easily fabricated. ${ }^{64,65}$ The use of fiducial markers or field edges to quantify patient setup errors can eliminate mechanical instability effects.

The reproducibility is established by checking both position (location and orientation of projected collimator axes) and linearity as the imager is repeatedly repositioned. This should also be performed at various gantry angles.

\section{Image quality}

Clinical image quality commissioning is based on spatial resolution and contrast resolution. All present day EPIDs provide $1 \%$ or better contrast resolution for larger objects $(>5 \mathrm{~mm})$. These characteristics are sufficient to perform portal localization on most radiotherapy fields. The Las Vegas phantom (Fig. 10) has been used in acceptance testing and continuing QA. It is composed of varying thickness and varying width holes embedded in aluminum which represent spatial and contrast resolution benchmarks. Visualizing a certain hole implies a specific resolution for a given linear accelerator/EPID combination. Properly setup EPIDs will typically be able to resolve the 17 shaded holes in Fig. 10 . Most should be able to resolve another four marked with X's. AMFPI systems should be able to resolve all the holes. Shalev and colleagues have introduced a phantom and soft- 
ware tool that allows the user to quantify EPID spatial resolution and contrast-to-noise ratio (CNR) ${ }^{66}$ The software determines CNR and spatial resolution from images acquired of a standardized phantom. The resolution and noise values reported may be used as baseline values for acceptance testing and ongoing QA of the EPID. The user is encouraged to demand this type of quality test at acceptance to help guarantee that the EPID is indeed operating at or above specifications. The spatial resolution indicated in the final row of Table II represents the spatial resolution (in line pairs per $\mathrm{mm}$ ) for commercial EPID configurations as determined using this phantom and analysis tool. ${ }^{66} \mathrm{~A}$ value of 0.25 indicates $2 \mathrm{~mm}$ spatial resolution. Regardless of which phantom is used and whether quantitative software is applied, the initial images represent baseline data for continuing quality assurance of the EPID. These should be the best images the system can obtain. In addition, images of anthropomorphic phantoms (phantoms used in a diagnostic radiology department may be better for this purpose than a sliced RANDO phantom) should be stored to represent the operation of the imager at optimum image quality.

\section{Software}

Commissioning of software involves testing of features such as EPID/linac control, network connections, storage, archival/retrieval and backup (including compression schemes), security functions, and analysis tools. During commissioning, responsibilities for these operations should be assigned.

If an EPID is intended for use in quantitative evaluation of patient setup, commissioning should involve measurement of known setup errors. These measurements should be designed to separate the results into those based on field placement and the location of the phantom in the field. The effects of image processing (e.g., image enhancement and edge detection) on the accuracy of setup analysis should be established. Image processing may affect the results of quantitative reporting. ${ }^{67}$

The commissioning process should include understanding and characterizing the limits of reference image generators (simulators, DRRs, etc.), since field placement errors are determined by comparing portal images to reference images.

A test should be performed to determine the ability of the system to reproduce a null transform on identical images. It is best to use the EPID's own software to compare an image to itself. A number of users should be recruited to use the setup verification tools to assess setup error on the image pair. This also allows the determination of inter- and intrauser variation in error detection, which should be established before setting correction thresholds. Typical accuracy for such tests have ranged from 0.5 to $2 \mathrm{~mm}$.

A second procedure involves attempting to assess a known transformation. In this case, a reference image of an anthropomorphic phantom can be taken. This image can be transformed by a known transformation, or the phantom can be moved by a known amount and reimaged. The measured transformation can then be compared to the expected trans-
TABLE IV. Frequency of QA tasks.

\begin{tabular}{|c|c|}
\hline Interval & $\begin{array}{c}\text { Task } \\
(\mathrm{P}-\text { physicist, } \mathrm{M}-\text { manufacturer, } \mathrm{E}-\text { engineer, } \\
\mathrm{T}-\text { therapist })\end{array}$ \\
\hline Daily & $\begin{array}{l}\text { Inspect imager housing }(\mathrm{T}) \\
\text { Test collision interlock }(\mathrm{T}) \\
\text { Acquire day's first image during machine warm-up } \\
\text { procedure to verify operation and image quality }(\mathrm{T}) \\
\text { Verify sufficient data capacity for day's images (P or } \\
\text { designate) }\end{array}$ \\
\hline Monthly & $\begin{array}{l}\text { Acquire image and inspect for artifacts }(\mathrm{P}) \\
\text { Perform constancy check of SNR, resolution and } \\
\quad \text { localization }(\mathrm{P}) \\
\text { Review image quality } \\
\text { Perform image and disk maintenance }(\mathrm{P}) \\
\text { Mechanical inspection [latches, collision sensors, optical } \\
\quad \text { components (P,E)] } \\
\text { Electrical connections (P,E) } \\
\text { Test collision interlock (P) } \\
\text { Hardcopy output (P) }\end{array}$ \\
\hline Annual & Perform full check of geometric localization accuracy $(\mathrm{P})$ \\
\hline
\end{tabular}

formation. Objective assessment of alignment tools can also be performed using a standard image data set. ${ }^{68}$

A complete dry run of a known phantom through the entire treatment process $(\mathrm{CT} / \mathrm{simulation}$, planning, reference image generation, initial setup, imaging, and setup measurement) allows testing of the proper operation of the EPID system within the confines of department infrastructure. This will allow identification of other potential sources of error, such as laser calibration differences or limits in DRR resolution. It is also advised to attempt to introduce errors into the alignment by rotating the phantom up to $6^{\circ}$ and by generating portal images of varying quality relative to a reference image. An accuracy of $\pm 3 \mathrm{~mm}$ and $\pm 1^{\circ}$ should be achievable. These tests should be performed for images acquired at all four cardinal gantry angles. Dry run procedures also help in training, education, and identifying individual responsibilities. Furthermore, the amount of time necessary for intended EPID use is indicated through dry runs.

\section{B. Quality assurance}

To maintain EPID performance, a quality assurance program must be put in place. The program must define specific measurements, frequencies, and tolerances (Table IV). Figure 11 shows examples of EPID QA daily and monthly worksheets for a matrix ion-chamber system in clinical use. The QA program should be in writing and records of the completed tasks should be kept for review.

Frequent (e.g., daily) quality assurance procedures include safety features such as mechanical integrity, collision interlocks, etc. Operational and image checks are accomplished by imaging a fixed phantom in a fixed geometry with a given dose. This allows rapid assessment of operability and image quality. 


\section{Daily EPID Quality Assurance Log \\ Dept. of Rad. Onc. \\ Initials:}

Date:

\begin{tabular}{|l|c|}
\hline \multicolumn{2}{|c|}{ Collision Interlocks } \\
\hline Upper Arm: Left Side Panel & $\square$ Alarm Sounds \\
\hline Upper Arm: Right Side Panel & $\square$ Alarm Sounds \\
\hline Collision Bar & $\square$ Alarm Sounds \\
\hline Cassette Head: All 4 Sides \& Top & $\square$ Alarm Sounds \\
\hline
\end{tabular}

\section{Image Quality}

1. Move EPID to P2, place Las Vegas Phantom on cassette surface, and align cross-hairs.

2. Close the jaws to the edge of the phantom.

3. Load patient "Physics - Daily QA" and choose the treatment field corresponding to the day of the week.

4. Acquire an image of the phantom.

5. Window and level the image until holes show up clearly.

6. In the diagram to the right, mark an $X$ in the right-hand circle of each row if it is visible in the image.

7. Save the image and record the following information:

\begin{tabular}{|l|cc|}
\hline Energy (circle one): & $6 \mathrm{MV}$ & $18 \mathrm{MV}$ \\
\hline Dose Rate (circle one): & $80 \mathrm{MU} / \mathrm{min} . \quad 400 \mathrm{MU} / \mathrm{min}$. \\
\hline Acquisition Mode: & Optimum Quality \\
\hline Specifications: & $\begin{array}{c}\text { Rows marked in diagram are visible } \\
\text { in the image for given energy. }\end{array}$ \\
\hline
\end{tabular}

\begin{tabular}{|c|c|c|}
\hline \multicolumn{3}{|c|}{ Position Verification } \\
\hline \multicolumn{3}{|c|}{$\begin{array}{l}\text { Move EPID to P2 ( } 138.5 \mathrm{~cm} \text { at cassette surface) } \\
\text { and record the following information: }\end{array}$} \\
\hline & $\begin{array}{l}\text { Optical Distance } \\
\text { Indicator }\end{array}$ & $\begin{array}{l}\text { Light-Field } \\
\text { Cross-Hairs }\end{array}$ \\
\hline \multicolumn{3}{|l|}{ Measured } \\
\hline Calibration & $138.5 \mathrm{~cm}$ & $0 \mathrm{~mm}$ \\
\hline \multicolumn{3}{|l|}{ Difference } \\
\hline Tolerance & $\pm 1 \mathrm{~cm}$ & $\pm 2 \mathrm{~mm}$ \\
\hline
\end{tabular}

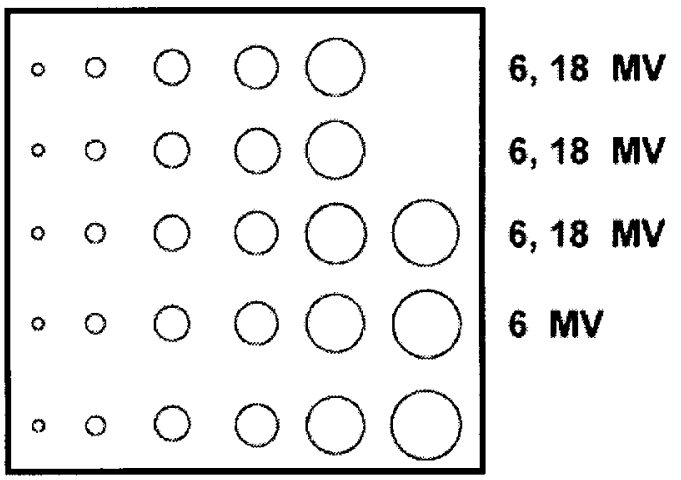

\begin{tabular}{|l|l|}
\hline \multicolumn{2}{|c|}{ Daily Problem Log } \\
\hline \multicolumn{1}{|c|}{ Problems } & Comments / Explanation / Description of Problem \\
\hline Deployment of R-Arm & \\
\hline Retraction of R-Arm & \\
\hline Interlocks & \\
\hline Image Quality & \\
\hline Software & \\
\hline Other & \\
\hline
\end{tabular}

(a)

FIG. 11. (a) Daily EPID QA worksheet sample; (b1) and (b2) monthly QA sample.

Monthly QA includes detailed safety and mechanical integrity checks (Table IV). A review of daily QA results to determine trends and degradation in image quality should be performed. The interval for recalibration of the imager is initially determined by the vendor and established at the time of commissioning, and may be changed through observation of trends in image quality. Periodic (e.g., monthly) disk and database maintenance should also be performed. 


\section{Monthly EPID Quality Assurance Log \\ Dept. of Rad. Onc.}

Date: Initials:

\begin{tabular}{|l|ll|l|l|l|}
\hline \multicolumn{6}{|c|}{ Review of Daily QA Log } \\
\hline Collision Interlocks & $\square$ No problems & $\square$ Problems & $\begin{array}{l}\text { If problems occurred, has action been taken and } \\
\text { documented in the Maintenance section? }\end{array}$ & $\square$ \\
\hline Position Verification & $\square$ No problems & $\square$ Problems & $\begin{array}{l}\text { If problems occurred, has the EPID been recali- } \\
\text { brated and documented in the Calibration section? }\end{array}$ & $\square$ Yes \\
\hline Image Quality & $\square$ No problems & $\square$ Problems & $\begin{array}{l}\text { If problems occurred, has the EPID been recali- } \\
\text { brated and documented in the Calibration section? }\end{array}$ & $\square$ Yes \\
\hline Daily Problem Log & $\square$ No problems & $\square$ Problems & $\begin{array}{l}\text { If problems occurred, have the problems been ad- } \\
\text { dressed and documented appropriately? }\end{array}$ & $\square$ Yes \\
\hline
\end{tabular}

\begin{tabular}{|l|c|c|c|c|}
\hline \multicolumn{3}{|c|}{ Collision Interlocks } \\
\hline Upper Arm: Left Side Panel & Alarm Sounds & $\begin{array}{c}\text { R-Arm Motion } \\
\text { Disabled }\end{array}$ & $\begin{array}{c}\text { Couch Motion } \\
\text { Disabled }\end{array}$ & $\begin{array}{c}\text { Gantry Motion } \\
\text { Disabled }\end{array}$ \\
\hline Upper Arm: Right Side Panel & $\square$ & & & \\
\hline Collision Bar & $\square$ & & \\
\hline Cassette Head: All 4 Sides \& Top & $\square$ & & \\
\hline
\end{tabular}

\begin{tabular}{|c|c|c|c|c|c|c|}
\hline \multicolumn{3}{|c|}{ Contrast and Spatial Resolution } & & & & \\
\hline \multicolumn{3}{|c|}{$\begin{array}{l}\text { 1. Move EPID to P2, place Las Vegas phantom on cassette surface, } \\
\text { and align cross-hairs. } \\
\text { 2. Close the jaws to the edge of the phantom (approx. } 11 \times 11 \mathrm{~cm} \text { ). } \\
\text { 3. Load patient "Physics - Monthly_QA" and choose the treatment field } \\
\text { corresponding to the desired energy / dose rate combination. } \\
\text { 4. Acquire an image of the phantom. } \\
\text { 5. Window and level the image until holes show up clearly. } \\
\text { 6. Save the images and record the information in the table below. } \\
\text { If the contrast resolution (determined by the rows visible) or the spatial } \\
\text { resolution (determined by the columns visible) do not meet tolerances, } \\
\text { image acquisition recalibration may be necessary. }\end{array}$} & $\begin{array}{ll}0 & 0 \\
0 & 0 \\
0 & 0 \\
0 & 0 \\
0 & 0\end{array}$ & & & $\mathbf{R z}$ \\
\hline Energy / Dose Rate & Row Visible & Row Tolerance & Column Visible & Colur & Tole & \\
\hline $6 \mathrm{MV} / 80 \mathrm{MU} / \mathrm{min}$ & & R4 & & & C5 & \\
\hline $6 \mathrm{MV} / 400 \mathrm{MU} / \mathrm{min}$ & & $\mathrm{R} 4$ & & & $\mathrm{C} 5$ & \\
\hline $18 \mathrm{MV} / 400 \mathrm{MU} / \mathrm{min}$. & & R3 & & & $\mathrm{C} 4$ & \\
\hline
\end{tabular}

(b)

FIG. 11. (Continued.) 


\section{Image Statistics}

1. Move EPID to P2 and set the collimator jaws to the edges of the detector.

2. Load patient "Physics - Monthly_QA" and choose the treatment field "Flood Field."

3. Acquire a flood field image.

4. When the image comes up, choose "View $\rightarrow$ Measure $\rightarrow$ Histogram".

5. Choose "ROI" to be "Full Image" and record the values found under "Pixel Statistics" below:

\begin{tabular}{|c|c|c|c|}
\hline & Max - Min & Mean & $N$ \\
\hline \multicolumn{4}{|l|}{ Measured } \\
\hline Tolerance & $<60$ & N/A & $<0.8 \%$ \\
\hline
\end{tabular}

\section{Position Verification}

Move EPID to P2 (138.5 cm at cassette surface) and record the following information:

\begin{tabular}{|l|c|c|}
\cline { 2 - 3 } & Optical Distance Indicator & Centering of Cross-Hairs \\
\hline Measured & & \\
\hline Accepted & $138.5 \mathrm{~cm}$ & $0 \mathrm{~mm}$ \\
\hline Tolerance & $\pm 1 \mathrm{~cm}$ & $\pm 2 \mathrm{~mm}$ \\
\hline
\end{tabular}

If either exceeds tolerance, conduct a mechanical recalibration and repeat position verification. Mechanical recalibration may also require image acquisition recalibration.

\begin{tabular}{|l|c|c|}
\hline \multicolumn{2}{|c|}{ Troubleshooting } & OK \\
\hline Horizontal Lines (thin): Consistent presence indicates need for recalibration. & \\
\hline Vertical Lines (thin): Consistent presence indicates faulty electrodes; replace detector. & $\square$ \\
\hline Dose Bars (thick horizontal): Consistent presence indicates linac dose instability. & $\square$ \\
\hline Bubbles: Indicates presence of air in chamber or compression of chamber; recalibrate. & $\square$ & $\square$ \\
\hline R-Arm Motion: Jerky motion might indicate imminent failure of motor or drive controller. & $\square$ \\
\hline Hand Pendant: Consistent loss of signal may indicate a failing battery. & $\square$ \\
\hline
\end{tabular}

\section{Calibration}

\begin{tabular}{|l|l|}
\hline$\square$ & Mechanical recalibration performed \& recorded in the "Maintenance" section. \\
\hline$\square$ & Image acquisition recalibration performed \& recorded in the "Calibration" section. \\
\hline
\end{tabular}

(c)

FIG. 11. (Continued.) 
A rapid check of software performance for quantitative measurement should be performed on an annual basis. This could involve a dry run using an anthropomorphic phantom, or could be performed using a geometric phantom (e.g., a radiosurgery target ball placed in a known location in the room coordinate system). Software QA should also be performed with upgrades and changes in the EPID system.

\section{CLINICAL APPLICATION OF EPIDs}

The primary applications of EPID include verification of patient setup and assessment of target and organ motion. Current research includes use of EPIDs for compensator design and verification, treatment machine QA, and patient dosimetry.

\section{A. Preparing for EPID implementation}

Certain specific goals and protocols for the use of EPIDs must be established before they can be successfully brought into the clinic. Table $\mathrm{V}$ lists examples of questions that should be discussed before EPID implementation. Table VI shows estimates of physician, therapist, and physicist time to implement a simple EPID program. It should be noted that EPID use and responsibilities differ between clinics around the world and between different EPIDs and these tables are guides indicative of issues each clinical team should address.

\section{B. Software tools}

The complexity of EPID software has evolved over the past decade in response to improved understanding of clinical applications as well as flexibility of acquisition modes for new EPID technology.

\section{Image acquisition}

A typical portal imaging system will have a user interface that allows selection of different image acquisition modes. Although the range of operating modes may vary, the following are commonly available on commercial EPIDs:

a. Single exposure (localization). In this mode of acquisition, a single image is acquired for a short period of time (typically at the start of the treatment). The duration of the exposure can either be controlled by a fixed time criterion or by the time that the beam is on.

b. Verification image. Verification images can either be an average of multiple images acquired during a period of treatment, or single images acquired over a longer period of time (higher dose) than the localization images mentioned previously.

c. Double exposure. This mode of operation is similar to that of weekly portal film acquisition. One image is the single exposure image, and the second is an "open field" image. Again, control of each image acquisition may be via fixed time intervals or by the duration of the beam. Typically, the open field and portal images are combined using a weighted sum to produce a single image. A field outline from the portal can also be automatically extracted and overlaid on the open field image. d. Movie loops. The digital nature of the EPID allows movie loops or on-line fluoroscopy to be acquired during treatment. In some cases, all of the images mentioned previously are generated by summation of one or more images acquired in a loop.

\section{Image enhancement tools}

Once an image has been acquired, unlike film, the image data can be manipulated to improve landmark visibility and image interpretation. Simple and sometimes automatic image enhancement tools are available on all EPIDs, giving a major advantage over film

One class of enhancement tools adjusts portal image contrast. The most basic of these, global contrast enhancement, involves manipulation of the gray scale lookup table of the video monitor displaying the image. The window and level values determine what pixel values are displayed and the range of video intensity values that these are mapped to. This method is typically interfaced to the user by "slider" bars adjacent to the image.

More advanced techniques employ nonlinear mappings of pixel values within the image based on redistributing intensity values to normalize the shape of the intensity histogram. These histogram equalization techniques alter a pixel's intensity based on the global or local adaptive histogram equalization (AHE) distribution of intensity. A disadvantage of AHE is the fact that the procedure is nonlinear, causing distortions of anatomical structures and field edges, which could affect quantitative measurement. ${ }^{69,70}$

High pass filtering can also achieve feature enhancement within a portal image, and can be performed by the convolution of a filter kernel and image to produce the feature enhanced image. ${ }^{71}$ Typical kernels include the Sobel (first derivative) and Laplacian (second derivative of the image) filters. A third known as an unsharp mask involves subtracting a smoothed version of the image from the original, removing all low frequency components. ${ }^{72}$ This processed image is combined in a weighted sum with the unprocessed image. Filtering can also be performed in the frequency domain by first calculating the Fourier transform of the image and applying a filter function to the image. ${ }^{73}$ Calculating the inverse transform of the result generates the filtered image.

A disadvantage of high pass filtering is the effect of noise amplification caused by the operation of the high pass filter. A compromise can be found by the application of a Weiner filter that produces the most optimal reconstruction of the image based on a least-squares minimization criterion. ${ }^{74}$ There is no single "best' enhancement scheme. Enhancement schemes should be selected by the users for the sites and image acquisition modes to be used clinically.

\section{Setup verification/error detection tools}

Treatment setup verification can be divided into verification of the geometric configuration of the treatment unit, and verification of the patient and target position with respect to the treatment geometry. 
TABLE V. Questions that are pertinent to implement an EPID for clinical use.

Questions Options

1. What is the purpose/goal of installing EPIDs in the clinic?

2. Which patients will EPID be used on for treatment verification?

3. How will the EPID be used?

4. What is the frequency of imaging?

4a. What image acquisition modes are available on the EPID?

5. What is the choice of reference image?

6. How will image evaluation be accomplished?

6a. How many review stations are needed and at what locations?

7. When will you intervene/adjust setup?

8. What image analysis protocol will be used?

8a. Which analysis tools are available and validated on the system?

9. How will physician approval be achieved?

9a. How will physician comments be communicated to others?

10. What are the resources needed for storage, archival, and retrieval?

10a. Is the system DICOM-RT compliant?

11. Implementation of a QA program

11a. What are the vendor established QA routines?

12. How will training and education for all users be scheduled? (a) Simple film replacement/routine QA

(b) Accurate and efficient patient setup and repositioning

(c) Assess random and systematic errors in treatment

(d) Assessment of the efficacy of immobilization techniques

(e) Inter- and intrafraction motion studies

(a) All patients?

(b) Special cases that are difficult to setup?

(c) Specific disease sites?

(a) Exclusively to eliminate film

(b) Combine with a predefined port film protocol

(a) Weekly

(b) Daily

(c) Dependent on site or patient

(d) Dependent on the statistics of setup error or decision rules

(a) Single exposure

(b) Double exposure

(c) Movie loops

(a) Digitally reconstructed radiograph

(b) Conventional simulation film

(c) First approved EPID image

(a) Electronically, side by side on computer workstation

(b) Hard copy on conventional view box

(a) At each treatment machine

(b) Also in viewing rooms

(c) Also in physicians offices

(a) Threshold for corrective action

(b) On-line-intrafraction correction

(c) Off-line-interfraction correction

(a) Visual inspection only

(b) Manual tools

(c) Semiautomated

(d) Automated

(a) Visual inspection only

(b) Manual tools

(c) Semiautomated

(d) Automated

(a) Signed hard copy off-line

(b) Electronic signature on-line

(c) Electronic signature off-line

(a) Hard copy

(b) Electronic annotation within EPID/information system

(c) Electronic email outside of EPID/information system

(A) Standalone hard disk

(b) Distributed database

(a) Establish baseline mechanical limits and imaging quality

(b) Establish daily and monthly protocol

(a) Establish training schedule

(b) Define personnel responsibilities

(c) Periodic in-service to ensure uniformly of clinical practice 
TABLE VI. Approximate personnel time commitments for various tasks related to the clinical use of an EPID.

\begin{tabular}{|c|c|c|c|c|c|}
\hline \multicolumn{2}{|c|}{ Task } & Time & per & Personnel & Comment \\
\hline \multicolumn{2}{|c|}{ Acceptance testing } & $1-2$ days & Installation & & Additional \\
\hline \multirow[t]{3}{*}{ Education } & Expert & 2 days + & Installation & Physicist & Per software \\
\hline & Therapist & 1 day & Installation & Therapist & \\
\hline & Physician & $\frac{1}{2}$ day & Installation & Physician & Revision \\
\hline \multicolumn{2}{|c|}{ Establish QA program } & $\frac{1}{2}$ day & Installation & Physicist & $\begin{array}{l}\text { Plus ongoing } \\
\text { monitoring }\end{array}$ \\
\hline \multirow[t]{2}{*}{ Operation } & Imaging & $<1-2 \min$ & Tx Field & Therapist & \\
\hline & Review & $0-5 \min$ & Tx Field & $\begin{array}{l}\text { Physician/ } \\
\text { Therapist }\end{array}$ & $\begin{array}{l}\text { Depends critically } \\
\text { on mode of use- } \\
\text { Table V }\end{array}$ \\
\hline \multirow[t]{3}{*}{ QA } & $\begin{array}{l}\text { Daily/ } \\
\text { Weekly }\end{array}$ & $3-5 \mathrm{~min}$ & Week & Therapist & \\
\hline & Monthly & $30 \mathrm{~min}$ & Month & Physicist & \\
\hline & Quarterly & $1-2 \mathrm{~h}$ & Quarter & Service & \\
\hline \multicolumn{2}{|c|}{$\begin{array}{l}\text { Commissioning Correction } \\
\text { Thresholds }\end{array}$} & 1 month & Protocol & All & $\begin{array}{l}\text { Software, intra- } \\
\text { and interuser, } \\
\text { etc. }\end{array}$ \\
\hline
\end{tabular}

Proper evaluation of treatment setup involves relating the information in a portal image to that extracted from a reference "gold standard" of treatment setup. The gold standard information can be a reference radiograph (simulation film or DRR), features extracted from the reference image (e.g., the field border and the anatomic landmark information), or three-dimensional models of the patient (e.g., CT data).

Digital measurement tools such as digital rulers can determine the distance from a given field border to critical projected anatomic interfaces. While not providing complete information on the nature of patient setup, such tools may be used for rapidly assessing critical features of daily setup such as field centering or spinal cord avoidance.

More detailed information about patient setup can be accomplished through the use of image registration algorithms. These can be classified loosely by the general mechanism used for selecting an optimal transformation.

Landmark-based techniques use geometric description of landmarks to determine a transformation that aligns a reference and portal image. Landmark descriptions that have been used include points, open curves, and drawn templates.

If points can be precisely localized, they can be aligned with high precision. The major difficulty with the use of point landmarks is the lack of suitable points. Observation of typical radiographs indicates very few internal anatomic regions that can be precisely localized as points on projections. Projections of external fiducial marks have been used as point landmarks, but these points may not properly reflect patient setup errors. Implanted fiducials have also been investigated for use in reproducible setup of the head. It is important to assess the reproducibility of selected point landmarks.

A large number of anatomic landmarks can be described adequately as open curve or line segments. A templatematching algorithm allows a user to draw an arbitrary set of landmarks on the reference image, and to determine an optimal transformation for alignment by manually shifting these landmarks until they are properly overlaid on the portal image. ${ }^{75}$ This system can be very fast, permitting on-line use. Other curve matching tools are more automated, providing the optimal transformation by determining the transform that best aligns overlapping curve segments. ${ }^{76}$

The use of landmark-based alignment algorithms requires a trained user to spend time to identify landmarks for use in alignment. Contrast-based algorithms show some promise for fully automated alignment. Typically, the intensity distribution in a region of a reference image is defined as a template. Using cross-correlation techniques, the transformation that optimally matches this template to a corresponding intensity distribution in the portal image is found.

Such techniques have been implemented to align whole images, ${ }^{77}$ and to select point landmarks based on the alignment of a series of small regions of interest containing distinct gray level distributions. ${ }^{78}$ An important consideration for contrast-based alignment techniques is the source of reference and setup radiographs. In order for most contrastbased algorithms to perform optimally, both images involved should have similar contrast distributions. Simulatorproduced images have different contrast than portal images due to the different absorption and scatter properties inherent with different energies of radiation. Solutions to this problem have been to establish a "gold standard" portal image at the beginning of treatment, or to optimize the DRR generation algorithms to produce contrast similar to that found in megavoltage radiographs..$^{68,79}$

Modern image processing techniques take advantage of the ability of computers to identify features in an image using properties similar to those a human observer is believed to use. Gilhuijs and colleagues developed a procedure for automated extraction of anatomic features and alignment to a 

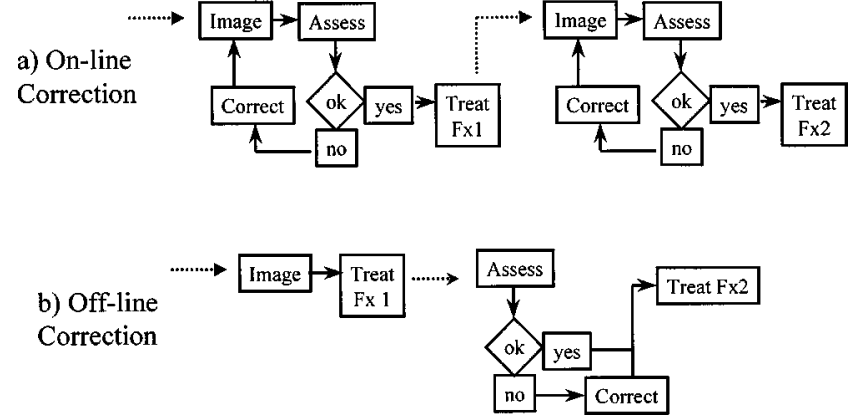

FIG. 12. Clinical imaging protocols with an EPID for (a) on-line and (b) off-line correction.

user-defined set of reference landmarks. ${ }^{80}$ In this procedure, a top hat transformation was used to extract a set of candidate coordinates for locations of bone-soft tissue interfaces. The optimal transformation that aligns the reference and portal anatomy is determined by chamfer matching of these coordinates with the distance space determined from the manually defined reference landmarks. Fritsch and colleagues have made significant progress in the application of computer vision techniques to the problem of image registration in radiotherapy. ${ }^{81}$ Using the multiscale medial axis filter, they have developed a system that extracts "cores" from radiographs. These cores are three-dimensional descriptions of features in images. Two of the dimensions are sued to indicate the location of object "middles," and the third describes the object's width at the given location. Such features have been evaluated for use in image alignment with promising results. ${ }^{80,81}$

Field edge detection is another important concern. There are two reasons to find the radiation field on the image. As most imagers do not maintain a rigid and reproducible relationship with respect to the central axis of the treatment unit, the location of the radiation field can be used to establish a coordinate system within which the variation of the location of patient anatomy can be determined. In the absence of a shaped radiation field, or when a field extends beyond the borders of the image, a graticule projection may also serve this purpose. A second important role for portal field border extraction is verification of the shape and orientation of the treatment portal.

A number of investigators have developed means of extracting the radiation field borders from portal images automatically. ${ }^{82-84}$ The intensity histogram from a portal image typically has two distinct peaks, representing the area outside the radiation field, and the pixels within the field. The range of pixel values between these peaks represents the beam penumbra. A reasonable threshold can be extracted automatically from this histogram to track the field borders. When a field border is in air, or near a rapidly changing density region of the patient, this technique may run into difficulty. Bijhold and colleagues developed a tracking algorithm that overcomes some of the limitations of thresholdbased field border extraction. ${ }^{82} \mathrm{McGee}$ developed a system to track the consistency of the field borders based on a model extracted from the initial treatment field. Wang and Fallone have developed a mathematical model for local field penumbra extraction. ${ }^{85}$

One significant limitation of much of the setup error analysis done to date is that the majority of clinical evaluation tools are based on two-dimensional analysis of portal images. An interactive procedure to quantify the setup variation of the patient in 3-D, based on fast computation of digitally reconstructed radiographs (DRRs) in two beam directions, has been developed. Computer aided comparison of these DRRs with corresponding portal images produces patient setup error information in $3 \mathrm{D} .{ }^{86-89}$

\section{EPID clinical use}

The types of errors detected include field and block shape errors and field or patient placement errors. There are two general methodologies in using an EPID for patient setup verification and correction; on-line or off-line (Fig. 12). For on-line correction, a pretreatment port can be acquired and evaluated such that any setup error will be corrected before the treatment continues. First day portal film localization is an example of an on-line correction.

The most basic manifestation of off-line correction occurs when the portal image is examined after treatment and, if necessary, a correction is made at the following treatment session. Standard weekly port films are an example of this strategy. Off-line correction has also evolved into strategies whereby multiple periodic images are evaluated to improve statistical certainty for one or more corrections over an entire treatment session.

\section{EPID clinical protocol (step by step)}

The following describes a simple procedure for using the EPID in the clinic. (1) For each patient, enter patient demographic, field data. Image acquisition data are also entered, e.g., single or double exposure, movie-loop, etc. The type and amount of data necessary varies depending on the EPID manufacturer. If the EPID is part of an integrated information system, much of the data input is done automatically when the treatment course is initially setup. (2) At treatment time, the EPID is put into imaging position, the patient is selected, the field is selected, and acquisition parameters loaded. Again, if the EPID becomes more integrated into the treatment system, the information system will automatically download the EPID with correct data for that patient and field. (3) Image the patient and take action as the protocol directs. The action may include doing nothing, performing on-line or off-line setup correction. If the EPID is part of the information system, recording, storing, and retrieving the image may be simplified.

\section{On-line EPID use}

An early group of on-line EPID studies involved taking prospective action based on a pretreatment port. This type of protocol has been implemented in a number of centers and allows the reduction of both random and systematic errors for each individual patient, but does not differentiate be- 


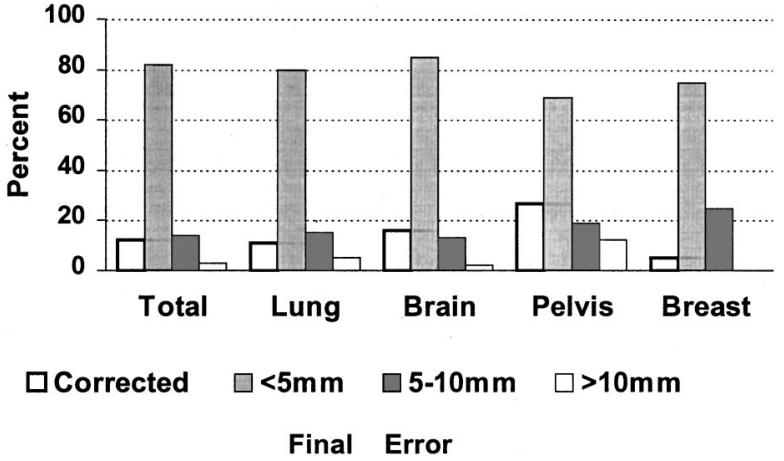

FIG. 13. Results of intrafractional corrections and final errors for various sites. The bar graph indicates how often corrections were made and what percentage of final errors were in each of three ranges. Modified from Ref. 17 with permission from Elsevier Science.

tween systematic and random error. ${ }^{9,17,18,90}$ Results of these studies indicate that up to $50 \%$ of initial fields are judged in error and corrected. The error correction rate is anatomical site dependent and due to the visual analysis, observer dependent as well. While improvement in setup accuracy was noted in these studies, final off-line analysis shows that some residual setup error remained. An example of on-line setup correction and final error is shown in Fig. $13 .{ }^{17}$ Visual analysis is not quantitative and as shown in Fig. 13, even after correction, quantitative off-line analysis found that $15 \%$ of setups were still in error by more than $5 \mathrm{~mm}$. In addition, these studies depend primarily on two-dimensional analysis and manual patient setup correction can increase treatment time. For these reasons, daily on-line EPID imaging is not practiced in many centers. There are, however, examples of on-line correction strategies in use today, where the clinicians feel that the additional time to make a correction is warranted..$^{91}$

More quantitative daily correction approaches have been developed, which utilize automated image analysis tools, developed commercially or in-house, to substantially increase accuracy, with modest increase in effort. A computer-aided on-line analysis and correction system has been implemented to correct pelvic and thoracic treatment setup errors daily. ${ }^{21,24}$ While these studies showed a significant improvement in setup accuracy, additional treatment time was required, due to the need to adjust patient setup. The computerized nature of the EPID allows it to be integrated into a larger scale decision-making system. Such an integrated system can help the users decide when it is appropriate to make a correction and when not to, based on the established physician and treatment planning guidelines. ${ }^{92}$ The quest for improved efficiency and automation in the use of EPIDs is ongoing and pursued both by research groups and vendors.

\section{Off-line EPID use}

Off-line EPID models can be separated into three groups, simple off-line correction (film model), monitoring, and statistical decision models.

a. Simple off-line. The simplest use of the EPID is to replace weekly portal filming, where the EPID is used to

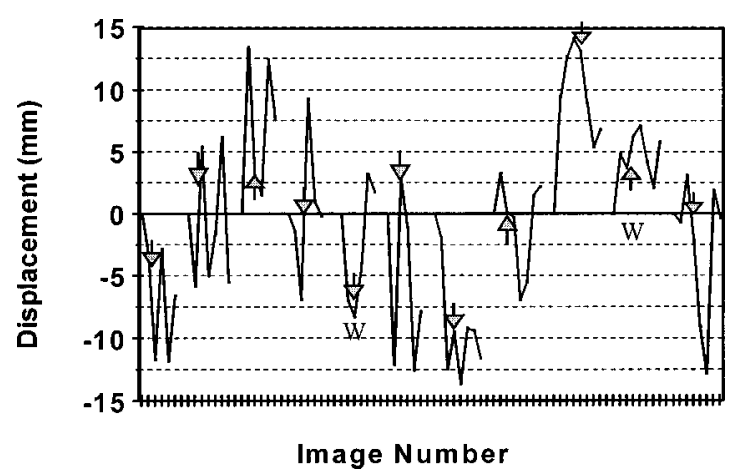

FIG. 14. Displacement results (in $\mathrm{mm}$ ) of the lung-chest wall interface measured during movie-loop imaging over multiple fractions. Daily imaging points are shown as arrows and weekly imaging as (W) (Ref. 98).

generate hard copy as with film [Fig. 12(b)]. The EPID also provides additional benefits compared to film; faster imaging time and image enhancement (e.g., contrast enhancement, edge enhancement) algorithms can be applied immediately. Error detection can be accomplished manually, with computer assistance in an interactive mode, or automatically.

b. Monitoring. The earliest clinical EPID studies were of the monitoring type, where images are acquired, but no action is taken. Lam described the frequency and magnitude of field placement errors (FPEs) in thoracic and abdominal radiotherapy, suggesting that errors exceeding conventional planning margins may not be uncommon. ${ }^{93}$ Others have created summary data showing the cumulative effect of daily FPEs on the course of radiotherapy for individual patients ${ }^{94}$ and then extended the methodology to indicate the effects of FPEs on treated doses. ${ }^{95}$ This strategy has also been utilized to determine time trends in patient setup accuracy, showing that patient setup error can increase during the course of therapy and that routine imaging is essential to maintain accurate treatment. $^{96}$

Movie loops have been used to monitor target and normal tissue motion between and during treatment fractions during tangential breast field treatment. ${ }^{7,16,97}$ The comprehensive analysis enabled by EPID use shows the magnitude and frequency of setup and motion errors for a group of patients and more importantly for individual patients. An example of motion of the lung-chest wall interface seen through six to seven images during each treatment fraction is shown in Fig. 14 , indicating the wide range of motion that occurs due to respiration during treatment. ${ }^{98}$ Daily and weekly imaging samples are also indicated in the figure. It is clear that weekly portal imaging cannot be used to quantify tissue motion due to respiration, which can exceed $2 \mathrm{~cm}$ during tangential breast treatments. ${ }^{22}$

Prostate motion studies using radio-opaque markers show that while the prostatic tissue relative to bony pelvis does not move appreciably during treatment, it can move over $1.5 \mathrm{~cm}$ relative to the bones between fractions. ${ }^{99}$ Other pelvic setup studies show that setup errors exceeding $1 \mathrm{~cm}$ were not uncommon, and that these intertreatment values exceed any intrafractional motion errors for the pelvis. ${ }^{100}$

c. Statistical models/decision rules. Statistical models 


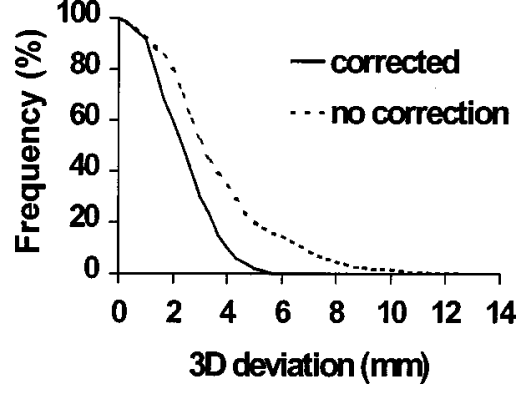

FIG. 15. Three-dimensional setup variation in Dutch prostate study (Ref. 29).

have evolved to allow treatment verification for complex treatments without a large increase in time or cost for the information. Two examples are presented.

Decision rule example 1 (analysis based on a global standard): A systematic error correction protocol based on establishing error thresholds derived from a patient population for a specific treatment site has been discussed. ${ }^{20,23,29}$ The need to correct systematic error for any patient is evaluated with respect to this institutional or global threshold. These studies have demonstrated that reduction of systematic error of approximately a factor of 2 (compared to uncorrected) is achievable, with an average of less than 10 images and approximately 0.5 corrections per patient treatment course (Fig. 15). In other words, with about the same imaging effort as film, and the tools of the EPID, significant error reduction can be achieved.

Decision rule example 2 (analysis based on an individual standard): The ability to gather enough data to make systematic and random error assessment on individual patients with EPID has also been introduced. In the population-based correction models, the setup errors are assessed for all patients [plotted in Fig. 16(a)]. If an EPID is used to acquire daily portal images for individual patients, then the data in Fig. 16(a) can be replotted in Fig. 16(b) as the average setup variation for each of 25 pelvic patients. Clearly, the margin of $11 \mathrm{~mm}$ (arrow) is unnecessarily large for an appreciable number of the patients, and yet inadequate for 2 patients. The

\section{MEAN $=5 \mathrm{~mm}, \mathrm{SD}=6 \mathrm{~mm}$}

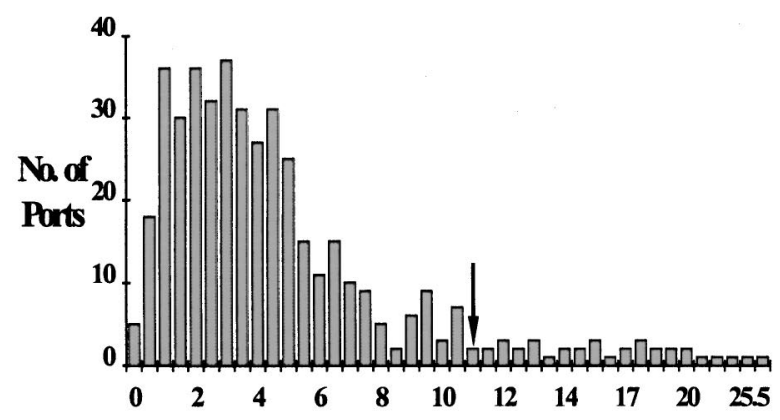

(a)

Shift of Iso-anter(m) data also suggest that some patients are highly reproducible in their daily setup. Individual treatment margins can be reoptimized for a specific margin reduction so that a higher dose might be delivered.

The concept of adaptive radiation therapy has been introduced by Yan et al. as a closed-loop feedback process for correction of the individual patient setup error. ${ }^{101,102}$ Extending the idea by Denham ${ }^{103}$ on the optimal frequency to take daily portal images, the nature of treatment variations are characterized for a few fractions early in the course of treatment such that they can be confidently estimated for the remaining course of treatment. This allows for the application of patient specific treatment corrections. Similar work in the use of EPID for early error detection and correction for dose escalation protocols is also underway. ${ }^{104}$

\section{Advanced applications}

\section{Treatment $Q A$}

The EPID has also been put to use for quality assurance of treatment machines ${ }^{64,105}$ and of treatment techniques, such as radiosurgery ${ }^{106}$ and dynamic treatment delivery. ${ }^{107-109}$ Investigators have used the EPID for the design ${ }^{110,111}$ and verification ${ }^{112}$ of compensating filters In each case, the EPID has allowed more precise, quantitative results to be obtained with much less effort than would have been achievable using conventional QA tools.

\section{Exit dosimetry}

More recently, there has been much interest in determining in vivo dose distributions during treatment with an EPID. While setup error and patient motion are quantified with EPID imaging, the ultimate value of concern is dose to target and normal tissue. Efforts to determine and quantify dose in two and three dimensions are under way. The earliest works investigated the characteristics of the various EPIDs for transmission dose measurement. ${ }^{65,113-116}$ These studies indicate that with the proper calibration and care, the EPID can be used to generate an exit dose image and values that are within $2 \%-5 \%$ of expected values. Additional work has

\section{Total Error}

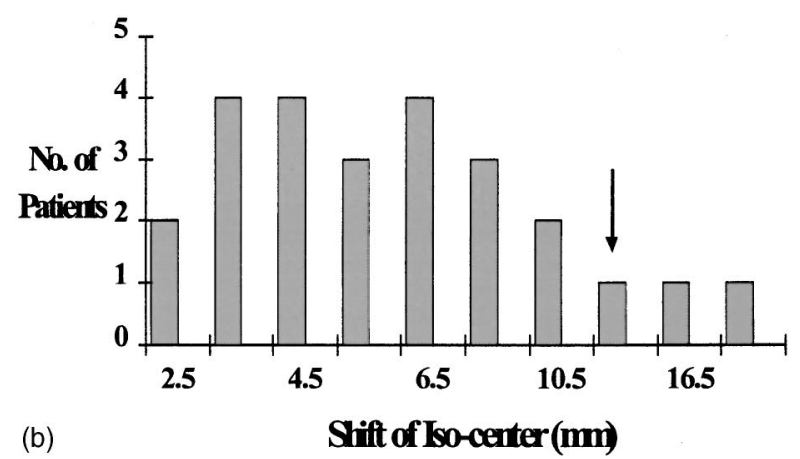

FIG. 16. (a) Margin (arrow) based on population statistics (b) replotted for individual patients, showing that the margin would not be optimum for all patients. 


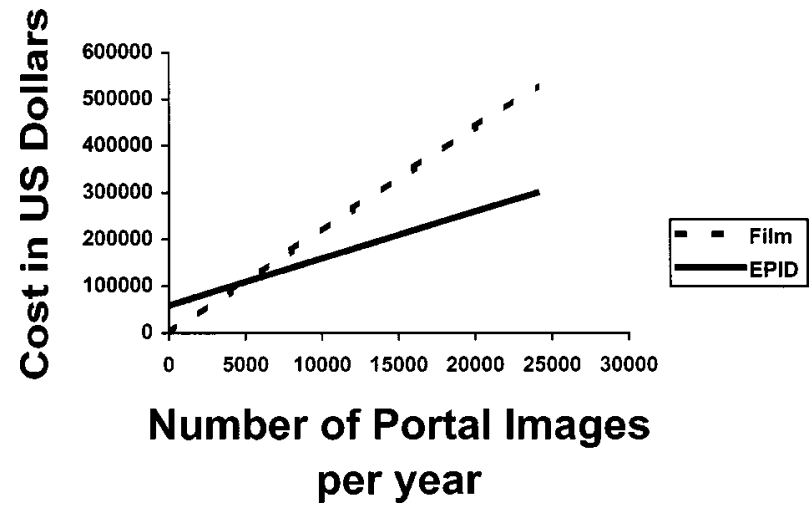

FIG. 17. Annual cost of portal imaging EPID and film vs imaging frequency.

gone into the interpretation of the EPID image in terms of a quantitative exit dose and implications for dose at the target. ${ }^{117,118}$ It should be noted that there may be significant differences related to quality control and calibration problems in determining dose with an EPID and extreme caution should be used.

\section{COST AND FUTURE}

\section{A. Cost}

The major expense for an EPID is the initial cost ranging from $\$ 80,000$ to $\$ 250,000$ (in 1999). The comparable initial expense for film portal imaging is about \$20,000. However, the ongoing costs for film portal imaging are substantial, where the EPID ongoing equipment and per image costs are almost negligible. The extra amount of time and labor needed to process film and display it for review is expensive, but varies depending on location and who performs the work. It has been shown that for large centers, or even smaller centers that image frequently, EPIDs can be more cost effective than film. ${ }^{119}$ It is therefore expected that with more frequent use, an EPID should be more economical than film. Figure 17 shows changes in total annual cost of imaging with film and EPID as a function of increasing use of portal imaging based on the usage model of a TG 58 member. The absolute numbers on each axis need to be adjusted for individual situations. The graph shows clearly that if portal imaging is performed frequently, then EPID is less expensive to use than film. Analyses such as these must consider capital costs, annual maintenance, and personnel time. It is important to note that this cost analysis treats EPID and film as identical in clinical value, ignoring the fact that the EPID is far more powerful than film for error analysis and in some cases can do things that cannot be done with film. Quantifying personnel costs specific to expected utilization of an EPID will help assess the economic impact of EPID use. The choice of correction strategy of any EPID protocol has the most direct impact on the allocation of personnel and computer resources. Table VII lists estimates of the necessary resources to implement various correction strategies. These estimates are based on imaging all patients at 2.5 fields per patient. Four strategies are distinguished. The first column pertains to the simplest strategy where images are inspected visually on-line to prevent gross error. The tolerance for correction should be set large so as not to impede treatment throughput but also to avoid erroneous correction. Images are not archived for analysis and the strategy incurs minimal cost. The second column presents perhaps the most complex and sophisticated use of the EPID where setup error is assessed and corrected on a daily basis. This strategy corrects for both systematic and random error, and in theory, should

TABLE VII. Estimates of personnel (FTF) and computer resources necessary for four correction strategies that can be used with an EPID, based on 2.5 treatment ports per patient.

\begin{tabular}{|c|c|c|c|c|}
\hline & $\begin{array}{l}\text { On-line } \\
\text { visual }\end{array}$ & $\begin{array}{l}\text { On-line } \\
\text { quantitative }\end{array}$ & $\begin{array}{c}\text { Off-line a } \\
\text { (weekly film model) }\end{array}$ & $\begin{array}{c}\text { Off-line b } \\
\text { (statistical } \\
\text { model) }\end{array}$ \\
\hline Tolerance (mm) & 7.0 & 2.0 & $3.0-5.0$ & 2.0 \\
\hline $\begin{array}{l}\text { Physics/computer } \\
\text { (FTE) }\end{array}$ & $0.1(\mathrm{QA})$ & $0.2(\mathrm{QA})$ & $\begin{array}{l}0.3 \\
(0.2 \text { QA, } 0.1 \text { support })\end{array}$ & 0.3 \\
\hline Operator $^{\mathrm{a}}(\mathrm{FTF})$ & $\begin{array}{l}0.05 \\
\text { (deploy) }\end{array}$ & $\begin{array}{l}0.10, \text { pre-Tx } \\
\text { localization }\end{array}$ & 0.05 & 0.05 \\
\hline Evaluator (FTE) & 0.02 & $\begin{array}{l}0.05 \text { (much } \\
\text { increased if } \\
\text { performed daily) }\end{array}$ & 0.05 & $0.3^{\mathrm{b}}$ \\
\hline $\begin{array}{l}\text { Software utility } \\
\text { beyond standard } \\
\text { image acquisition } \\
\text { software }\end{array}$ & 0 & $\begin{array}{l}\text { Quantitative } \\
\text { measurement tools }\end{array}$ & $\begin{array}{l}\text { Quantitative } \\
\text { measurement tools, } \\
\text { annotation }\end{array}$ & $\begin{array}{l}\text { Quantitative } \\
\text { measurement } \\
\text { tools, } \\
\text { annotation, } \\
\text { statistical } \\
\text { model }\end{array}$ \\
\hline Hardware & 0 & $\begin{array}{l}\text { Reference image } \\
\text { import, } \\
\text { record of event }\end{array}$ & $\begin{array}{l}\text { Review station, } \\
\text { Reference image } \\
\text { import, (hardcopy?) }\end{array}$ & $\begin{array}{l}\text { Analysis } \\
\text { station, } \\
\text { reference } \\
\text { image import }\end{array}$ \\
\hline
\end{tabular}

${ }^{a}$ Operator indicates treatment machine time.

${ }^{\mathrm{b}}$ Includes error analysis and statistical model/decision rule analysis for all patients. 
achieve the highest accuracy. However, this strategy can also be most costly in terms of time and effort. The third column is for an off-line correction strategy that is similar to the weekly port film practice. Additional software and hardware options can be very helpful. The final column represents the off-line strategy that is based on statistical decision models. The approach requires the commitment of personnel and computer resources to archive and analyze the data, but also provides the potential for reduction of effort at the treatment unit. For example, field placement error can be corrected by moving a multileaf collimator via network. Note that the associated resource costs stated for the four strategies are estimates of total cost. This should be compared to film imaging [similar to column off-line (a)]. For larger departments, some economy of scale should be expected.

\section{B. Future}

Modern technology is yielding new flat panel AMFPI radiographic detectors. These devices take two forms: photodiode arrays (e.g., amorphous silicon arrays) $)^{52-54,120-122}$ and photoconductor arrays (e.g., amorphous selenium arrays). ${ }^{55-61,123}$ These devices show promise to yield image quality superior to film, with all the power of an EPID. The amorphous silicon arrays have excellent imaging characteristics. Measurements have shown that these devices are x-ray quantum limited, ${ }^{124}$ while other reports suggest that, apart from the finite pixel size, the amorphous silicon array light sensor has a negligible effect on spatial resolution of the EPID. ${ }^{125-127}$ These detectors are capable of providing verification for advanced treatment delivery systems such as those used in IMRT.

New technology applied to setup and portal verification is also being developed. The approaches include the use of kilovoltage X-ray beams, ${ }^{128-131}$ the use of video camera positioning systems, ${ }^{132,133}$ and ultrasound. ${ }^{134-138}$

It is very important to note that the data infrastructure and the clinical utilization process must be understood to fully take advantage of any new or advanced technology.

\section{SUMMARY AND RECOMMENDATIONS}

The TG 58 report was written to enhance the knowledge of the medical physicist in implementing EPID technology in the clinic. From reading this report, the reader should understand the following.

(1) The basic physical principles of image formation and megavoltage imaging. This provides the reader with the essential background necessary to understand the function, limitations, and quality assurance of EPID systems.

(2) The technical and practical manifestations of the megavoltage imagers. This gives the reader an understanding of the hardware, software, and production characteristics of commercially available systems. Critical components related to image quality, EPID operation, performance, and safety are indicated and must be understood for optimal clinical use.

(3) Details of installation, commissioning, and developing and performing continuing quality assurance. Essential char- acteristics to consider and steps to be taken to bring EPID technology into the clinic, verify proper operation, and establish a viable quality assurance program are provided.

(4) Critical questions that should be discussed to help the reader prepare for the purchase, installation, and continuing effective use of an EPID. This includes understanding the clinical situation and potential resource commitments.

(5) Availability and operation of various image acquisition, enhancement, and analysis software to make appropriate equipment selection and specifications.

(6) The models of successful clinical EPID use, which demonstrate a wide variety of application from simple to sophisticated. Reading these references provides detailed information on the cost, benefit, and implementation of each EPID protocol.

(7) How to establish costs for EPID implementation, from capital equipment to human resources. Choice of clinical application has a strong influence on total costs.

(8) That the technology for EPID is changing and improving. Any purchase should consider upgrades in both software and hardware.

This task group recommends that:

(i) The medical physicist become familiar with the physics of megavoltage portal imaging (Sec. II) and its commercial manifestations (Sec. III). This information allows the reader to establish clear specifications and to maintain an effective EPID.

(ii) The medical physicist understand the details of the installation, commissioning, and the QA process of an EPID (Sec. IV). Only if these issues are understood can the physicist be prepared to bring the EPID system into the clinic and maintain the system at optimum performance.

(iii) The treatment team evaluate the tables in Sec. V regarding clinical use, resource commitment, and education. Since each clinical use of EPID may be different, evaluating these issues before selecting and implementing an EPID is paramount.

(iv) The medical physicist evaluate ongoing education, upgrades, and clinical uses of EPID to remain knowledgeable in maintaining and improving the quality of EPID application.

\section{ACKNOWLEDGMENTS}

The authors wish to thank all of our clinical colleagues that have provided input and discussion on the use of EPID.

\footnotetext{
${ }^{\text {a) }}$ Author to whom all correspondence should be addressed; electronic mail: herman.michael@mayo.edu

${ }^{1}$ J. E. Marks, A. G. Haus, H. G. Sutton, and M. L. Griem, "Localization error in the radiotherapy of Hodgkin's disease and malignant lymphoma with extended mantle fields," Cancer (N.Y.) 34, 83-90 (1974).

${ }^{2}$ I. Rabinowitz, J. Broomberg, M. Goitein, K. McCarthy, and J. Leong, "Accuracy of radiation field alignment in clinical practice," Int. J. Radiat. Oncol., Biol., Phys. 11, 1857-67 (1985).

${ }^{3}$ S. A. Rosenthal, J. M. Galvin, J. W. Goldwein, A. R. Smith, and P. H. Blitzer, "Improved methods for determination of variability in patient positioning for radiation therapy using simulation and serial portal film measurements," Int. J. Radiat. Oncol., Biol., Phys. 23, 621-625 (1992).
} 
${ }^{4}$ J. M. Balter, H. M. Sandler, K. Lam, R. L. Bree, A. S. Lichter, and R. K. ten Haken, "Measurement of prostate movement over the course of routine radiotherapy using implanted markers," Int. J. Radiat. Oncol., Biol., Phys. 31, 113-8 (1995).

${ }^{5}$ S. E. Griffiths, R. G. Pearcey, and J. Thorogood, "Quality control in radiotherapy: The reduction of field placement errors," Int. J. Radiat. Oncol., Biol., Phys. 13, 1583-1588 (1987).

${ }^{6}$ H. Huizenga, P. C. Levendag, P. M. De Porre, and A. G. Visser, “Accuracy in radiation field alignment in head and neck cancer: A prospective study," Radiother. Oncol. 11, 184-187 (1988).

${ }^{7}$ G. van Tienhoven, J. H. Lanson, D. Crabeels, S. Heukelom, and B. J. Mijnheer, "Accuracy in tangential breast treatment set-up: A portal imaging study," Radiother. Oncol. 22, 317-322 (1991).

${ }^{8}$ C. Mitine, A. Dutreix, and E. van der Schueren, "Tangential breast irradiation: Influence of technique of set-up on transfer errors and reproducibility," Radiother. Oncol. 22, 308-310 (1991).

${ }^{9}$ A. Ezz, P. Munro, A. T. Porter, J. Battista, D. A. Jaffray, A. Fenster, and S. Osborne, "Daily monitoring and correction of radiation field placement using a video-based portal imaging system: A pilot study," Int. J. Radiat. Oncol., Biol., Phys. 22, 159-165 (1992).

${ }^{10}$ J. Bijhold, J. V. Lebesque, A. A. Hart, and R. E. Vijlbrief, “'Maximizing setup accuracy using portal images as applied to a conformal boost technique for prostatic cancer,"' Radiother. Oncol. 24, 261-271 (1992).

${ }^{11}$ C. Weltens, G. Leunens, A. Dutreix, J. Cosset, F. Eschwege, and E. van der Schueren, "Accuracy in mantle field irradiations: Irradiated volume and daily dose," Radiother. Oncol. 29, 18-26 (1993).

${ }^{12}$ C. Mitine, A. Dutreix, and E. van der Schueren, "Black and white in accuracy assessment of megavoltage images: The medical decision is often gray," Radiother. Oncol. 28, 31-36 (1993).

${ }^{13}$ P. B. Dunscombe, K. Fox, S. Loose, and K. Leszczynski, “The investigation and rectification of field placement errors in the delivery of complex head and neck fields," Int. J. Radiat. Oncol., Biol., Phys. 26, 155161 (1993).

${ }^{14}$ P. Dunscombe, S. Loose, and K. Leszczynski, "'Sizes and sources of field placement error in routine irradiation for prostate cancer," Radiother. Oncol. 26, 174-176 (1993).

${ }^{15}$ M. A. Hunt, G. J. Kutcher, C. Burman, D. Fass, L. Harrison, S. Leibel, and Z. Fuks, "The effect of setup uncertainties on the treatment of nasopharynx cancer,' Int. J. Radiat. Oncol., Biol., Phys. 27, 437-447 (1993).

${ }^{16}$ C. L. Creutzberg, V. G. Althof, H. Huizenga, A. G. Visser, and P. C. Levendag, "Quality assurance using portal imaging: The accuracy of patient positioning in irradiation of breast cancer,' Int. J. Radiat. Oncol., Biol., Phys. 25(3), 529-539 (1993).

${ }^{17}$ M. G. Herman, R. A. Abrams, and R. R. Mayer, "Clinical use of on-line portal imaging for daily patient treatment verification,' Int. J. Radiat. Oncol., Biol., Phys. 28(4), 1017-1023 (1994).

${ }^{18}$ J. Gildersleve, D. P. Dearnaley, P. M. Evans, M. Law, C. Rawlings, and W. Swindell, "A randomised trial of patient repositioning during radiotherapy using a megavoltage imaging system," Radiother. Oncol. 31, 161-168 (1994).

${ }^{19}$ M. A. Hunt, T. E. Schultheiss, G. E. Desobry, M. Hakki, and G. E. Hanks, "An evaluation of setup uncertainties for patients treated to pelvic sites,' Int. J. Radiat. Oncol., Biol., Phys. 32, 227-233 (1995).

${ }^{20}$ A. Bel, R. Keus, R. E. Vijlbrief, and J. V. Lebesque, "Setup deviations in wedged pair irradiation of parotid gland and tonsillar tumors, measured with an electronic portal imaging device," Radiother. Oncol. 37, 153159 (1995).

${ }^{21}$ F. Van den Heuvel, W. De Neve, D. Verellen, M. Coghe, V. Coen, and G. Storme, "Clinical implementation of an objective computer-aided protocol for intervention in intra-treatment correction using electronic portal imaging," Radiother. Oncol. 35, 232-239 (1995).

${ }^{22}$ A. Lirette, J. Pouliot, M. Aubin, and M. Larochelle, "The role of electronic portal imaging in tangential breast irradiation: A prospective study," Radiother. Oncol. 37, 241-245 (1995).

${ }^{23}$ A. Bel, P. H. Vos, P. T. Rodrigus, C. L. Creutzberg, A. G. Visser, J. C. Stroom, and J. V. Lebesque, "High-precision prostate cancer irradiation by clinical application of an offline patient setup verification procedure, using portal imaging," Int. J. Radiat. Oncol., Biol., Phys. 35, 321-332 (1996).

${ }^{24}$ J. Van de Steene, F. Van den Heuvel, A. Bel, D. Verellen, J. De Mey, M. Noppen, M. De Beukeleer, and G. Storme, "Electronic portal imaging with on-line correction of setup error in thoracic irradiation: Clinical evaluation,' Int. J. Radiat. Oncol., Biol., Phys. 40, 967-976 (1998).

${ }^{25}$ J. E. Marks, A. G. Haus, H. G. Sutton, and M. L. Griem, "The value of frequent treatment (N.Y.) verification films in reducing localization error in the irradiation of complex fields," Cancer (N.Y.) 37, 2755-2761 (1976).

${ }^{26}$ S. C. Taborsky, W. C. Lam, R. E. Sterner, and G. M. Skarda, "'Digital imaging for radiation therapy verification," Proc. SPIE 314, 164-171 (1981).

${ }^{27}$ N. A. Baily, R. A. Horn, and T. D. Kampp, "Fluoroscopic visualization of megavoltage therapeutic x ray beams," Int. J. Radiat. Oncol., Biol., Phys. 6, 935-939 (1980).

${ }^{28}$ A. Bel, M. van Herk, and J. V. Lebesque, "Target margins for random geometrical treatment uncertainties in conformal radiotherapy," Med. Phys. 23, 1537-1545 (1996).

${ }^{29}$ J. V. Lebesque, P. Remeijer, L. van Riel, P. V. Vos, J. C. J. de Boer, A. G. Visser, E. van Lin, and S. Feenstra, "Clinical evaluation of setup verification and correction protocols: Results of Multicentre Studies of the Dutch Cooperative EPID Group," The Fifth International Electronic Portal Imaging Workshop, Phoenix, AZ, 1998, p. 20.

${ }^{30}$ J. W. Motz and M. Danos, "Image information content and patient exposure," Med. Phys. 5, 8-22 (1978).

${ }^{31}$ D. W. Rogers, "Fluence to dose equivalent conversion factors calculated with EGS3 for electrons from $100 \mathrm{keV}$ to $20 \mathrm{GeV}$ and photons from 11 keV to 20 GeV," Health Phys. 46, 891-914 (1984).

${ }^{32}$ K. Doi, G. Holje, L.-N. Loo, H.-P. Chan, J. M. Sandrik, R. J. Jennings, and R. F. Wagner, "MTF's and Wiener Spectra of Radiographic ScreenFilm Systems,"' Bureau of Radiological Health, HHS Publ. No. 82, 1982, pp. $81-87$.

${ }^{33}$ B. Wowk and S. Shalev, "Thick phosphor screens for on-line portal imaging,"' Med. Phys. 21, 1269-1276 (1994).

${ }^{34} \mathrm{R}$. Swank, "Measurement of absorption and noise in an x-ray image intensifier," J. Appl. Phys. 45, 3673-3678 (1974).

${ }^{35}$ P. Munro, "Imaging with high energy radiation beams," Ph.D. thesis, 1990.

${ }^{36}$ P. Munro, J. A. Rawlinson, and A. Fenster, “Therapy imaging: A signalto-noise analysis of a fluoroscopic imaging system for radiotherapy localization," Med. Phys. 17, 763-772 (1990).

${ }^{37}$ P. Munro, J. A. Rawlinson, and A. Fenster, “Therapy imaging: A signalto-noise analysis of metal plate/film detectors," Med. Phys. 14, 975-984 (1987).

${ }^{38}$ J. P. Bissonnette, I. A. Cunningham, D. A. Jaffray, A. Fenster, and P. Munro, "A quantum accounting and detective quantum efficiency analysis for video-based portal imaging," Med. Phys. 24, 815-826 (1997).

${ }^{39}$ P. Munro, J. A. Rawlinson, and A. Fenster, "A digital fluoroscopic imaging device for radiotherapy localization," Int. J. Radiat. Oncol., Biol., Phys. 18, 641-649 (1990).

${ }^{40}$ M. Ishida, K. Doi, L. N. Loo, C. E. Metz, and J. L. Lehr, “Digital image processing: Effect on detectability of simulated low-contrast radiographic patterns,"' Radiology 150, 569-575 (1984).

${ }^{41}$ R. T. Droege, "A megavoltage MTF measurement technique for metal screen-film detectors," Med. Phys. 6, 272-279 (1979).

${ }^{42}$ P. Munro, J. A. Rawlinson, and A. Fenster, "Therapy imaging: Limitations of imaging with high energy x-ray beams," Proc. SPIE 767, 178184 (1987)

${ }^{43} \mathrm{M}$. van Herk and $\mathrm{H}$. Meertens, "A matrix ionization chamber imaging device for on-line patient setup verification during radiotherapy," Radiother. Oncol. 11(4), 369-378 (1988).

${ }^{44}$ B. Wowk, T. Radcliffe, K. W. Leszczynski, S. Shalev, and R. Rajapakshe, "Optimization of metal/phosphor screens for on-line portal imaging," Med. Phys. 21, 227-235 (1994).

${ }^{45}$ W. Swindell, E. J. Morton, P. M. Evans, and D. G. Lewis, "The design of megavoltage projection imaging systems: Some theoretical aspects," Med. Phys. 18, 855-866 (1991).

${ }^{46}$ J. P. Bissonnette, D. A. Jaffray, A. Fenster, and P. Munro, "Optimal radiographic magnification for portal imaging," Med. Phys. 21, 14351445 (1994).

${ }^{47}$ D. A. Jaffray, J. J. Battista, A. Fenster, and P. Munro, "X-ray scatter in megavoltage transmission radiography: Physical characteristics and influence on image quality,"' Med. Phys. 21, 45-60 (1994).

${ }^{48}$ A. L. Boyer, L. Antonuk, A. Fenster, M. Van Herk, H. Meertens, P. Munro, L. E. Reinstein, and J. Wong, "A review of electronic portal imaging devices (EPIDs)," Med. Phys. 19, 1-16 (1992). 
${ }^{49} \mathrm{~S}$. Webb, The Physics of Three Dimensional Radiation Therapy, First ed. (IOP, Bristol, 1993).

${ }^{50} \mathrm{H}$. Roehrig and C. Cheng, "Real-time imaging detectors for portal imaging,'” Proc. SPIE 2009, 144-167 (1993).

${ }^{51}$ P. Munro, "Portal imaging technology: Past, present and future.," Semin. Rad. Oncol. 5, 115-133 (1995).

${ }^{52}$ L. E. Antonuk, J. Boudry, J. Yorkston, C. F. Wild, M. J. Longo, and R. A. Street, "Radiation-damage studies of amorphous-silicon photodiiode sensors for applications in radiotherapy x-ray imaging," Nucl. Instrum. Methods Phys. Res. A 299, 143-146 (1990).

${ }^{53}$ L. E. Antonuk, J. Boudry, W. Huang, D. L. McShan, E. J. Morton, J. Yorkston, M. J. Longo, and R. A. Street, "'Demonstration of megavoltage and diagnostic $\mathrm{x}$-ray imaging with hydrogenated amorphous silicon arrays [published erratum appears in Med. Phys. 20, 825 (1993)]," Med. Phys. 19, 1455-1466 (1992).

${ }^{54}$ L. E. Antonuk, J. Boudry, Y. El-Mohri, W. Huang, J. H. Siewerdsen, J. Yorkston, and R. A. Street, "A high resolution, high frame rate, flatpanel TFT array for digital x-ray imaging," Proc. SPIE 2163, 118-128 (1994).

${ }^{55} \mathrm{~W}$. Zhao and J. A. Rowlands, "Large-area solid state detector for radiology using amorphous selenium," Proc. SPIE 1651, 134-143 (1992).

${ }^{56} \mathrm{~W}$. Zhao and J. A. Rowlands, "'Digital radiology using self-scanned readout of amorphous selenium," Proc. SPIE 1896, 114-120 (1993).

${ }^{57} \mathrm{~W}$. Zhao and J. A. Rowlands, "X-ray imaging using amorphous selenium: Feasibility of a flat panel self-scanned detector for digital radiology," Med. Phys. 22, 1595-1604 (1995).

${ }^{58}$ W. Zhao, J. Rowlands, A. S. Germann, D. Waechter, and Z. J. Huang, "Digital radiology using self-scanned readout of amorphous selenium: Design considerations for mammography," Proc. SPIE 2432, 250-259 (1995).

${ }^{59}$ W. Zhao, I. Blevis, A. S. Germann, J. Rowlands, D. Waechter, and Z. J. Huang, "Flat panel detector for digital radiology using active matrix readout of amorphous selenium," Proc. SPIE 2708, 523-531 (1996).

${ }^{60} \mathrm{H}$. Wang, T. Falco, and B. G. Fallone, "A metal screen-amorphous selenium based image receptor in megavoltage portal imaging," Med. Phys. 23, 1130 (1996).

${ }^{61}$ D. Mah, J. A. Rowlands, and J. A. Rawlinson, "Portal imaging with amorphous selenium: Demonstration of image quality using a photoinduced discharge approach," Med. Phys. 23, 1031 (1996).

${ }^{62} \mathrm{H}$. Meertens, M. van Herk, and J. Weeda, "A liquid ionization detector for digital radiography of therapeutic megavoltage photon beams," Phys. Med. Biol. 30, 313-321 (1985).

${ }^{63}$ M. van Herk, W. Fencl, and A. van Dalen, "Design and implementation of a high speed matrix ionization chamber system," Med. Phys. 22, 991 (1995).

${ }^{64}$ J. Balter, A. Thompson, and R. K. Ten Haken, “Automated quality assurance of mechanical components of a computer controlled accelerator using and EPID," Fourth International Workshop on Electronic Portal Imaging, Amsterdam, 1996.

${ }^{65}$ Y. Zhu, X. Q. Jiang, and J. Van Dyk, "Portal dosimetry using a liquid ion chamber matrix: Dose response studies,'” Med. Phys. 22, 1101-1106 (1995).

${ }^{66}$ R. Rajapakshe, K. Luchka, and S. Shalev, "A quality control test for electronic portal imaging devices," Med. Phys. 23, 1237-1244 (1996).

${ }^{67}$ D. Verellen, W. De Neve, F. Van den Heuvel, M. Coghe, O. Louis, and G. Storme, "On-line portal imaging: Image quality defining parameters for pelvic fields-a clinical evaluation," Int. J. Radiat. Oncol., Biol., Phys. 27(4), 945-952 (1993).

${ }^{68}$ D. S. Fritsch, S. Raghavan, A. Boxwala, J. Earnhart, G. Tracton, T. Cullip, and E. L. Chaney, "Benchmark test cases for evaluation of computer-based methods for detection of setup errors: Realistic digitally reconstructed electronic portal images with known setup errors,"' Int. J. Radiat. Oncol., Biol., Phys. 37, 199-204 (1997).

${ }^{69} \mathrm{~K}$. W. Leszczynski, S. Shalev, and S. Ryder, "A study on the efficacy of digital enhancement of on-line portal images [published erratum appears in Med. Phys. 20, 255 (1993)]," Med. Phys. 19(4), 999-1005 (1992).

${ }^{70}$ J. M. Balter, C. A. Pelizzari, and G. T. Chen, "ROC evaluation of digital enhancement techniques for portal images,"’ Med. Phys. 16, 455 (1989).

${ }^{71}$ W. K. Pratt, Digital Image Processing (Wiley, New York, 1978).

${ }^{72}$ C. E. Metz, H. P. Chan, K. Doi, and J. H. Shen, "Contrast enhancement of noisy images by windowing: Limitations due to the finite dynamic range of the display system," Med. Phys. 16, 170-178 (1989).
${ }^{73}$ R. N. Bracewell, Two-dimensional Imaging (Prentice-Hall, Englewood Cliffs, NJ, 1995).

${ }^{74} \mathrm{H}$. Meertens, M. van Herk, and J. Weeda, "An inverse filter for digital restoration of portal images," Phys. Med. Biol. 33, 687-702 (1988).

${ }^{75} \mathrm{P}$. H. Vos, "On-line repositioning based on portal imaging in clinical routine," Fifth international Workshop on Electronic Portal Imaging, Phoenix, AZ, 1998, p. 45.

${ }^{76}$ J. M. Balter, C. A. Pelizzari, and G. T. Chen, "Correlation of projection radiographs in radiation therapy using open curve segments and points," Med. Phys. 19, 329-334 (1992).

${ }^{77}$ S. M. Jones and A. L. Boyer, "Investigation of an FFT-based correlation technique for verification of radiation treatment setup," Med. Phys. 18, 1116-1125 (1991)

${ }^{78}$ J. Moseley and P. Munro, "A semiautomatic method for registration of portal images," Med. Phys. 21, 551-558 (1994).

${ }^{79}$ L. Dong and A. L. Boyer, "A portal image alignment and patient setup verification procedure using moments and correlation techniques," Phys. Med. Biol. 41, 697-723 (1996).

${ }^{80}$ K. G. Gilhuijs, A. Touw, M. van Herk, and R. E. Vijlbrief, "'Optimization of automatic portal image analysis," Med. Phys. 22, 1089-1099 (1995).

${ }^{81}$ D. S. Fritsch, E. L. Chaney, A. Boxwala, M. J. McAuliffe, S. Raghavan, A. Thall, and J. R. Earnhart, "Core-based portal image registration for automatic radiotherapy treatment verification," Int. J. Radiat. Oncol., Biol., Phys. 33, 1287-300 (1995).

${ }^{82}$ J. Bijhold, M. van Herk, R. Vijlbrief, and J. V. Lebesque, "Fast evaluation of patient set-up during radiotherapy by aligning features in portal and simulator images," Phys. Med. Biol. 36, 1665-1679 (1991).

${ }^{83} \mathrm{~K}$. Eilertsen, "Automatic detection of single MLC leaf positions with corrections for penumbral effects and portal imager dose rate characteristics," Phys. Med. Biol. 42, 313-334 (1997).

${ }^{84} \mathrm{H}$. Wang and B. G. Fallone, "A robust morphological algorithm for automatic radiation field extraction and correlation of portal images," Med. Phys. 21, 237-244 (1994).

${ }^{85} \mathrm{H}$. Wang and B. G. Fallone, "A mathematical model of radiation field edge localization,” Med. Phys. 22, 1107-1110 (1995).

${ }^{86}$ K. G. Gilhuijs, K. Drukker, A. Touw, P. J. van de Ven, and M. van Herk, "Interactive three dimensional inspection of patient setup in radiation therapy using digital portal images and computed tomography data," Int. J. Radiat. Oncol., Biol., Phys. 34(4), 873-885 (1996).

${ }^{87}$ K. G. Gilhuijs, P. J. van de Ven, and M. van Herk, "Automatic threedimensional inspection of patient setup in radiation therapy using portal images, simulator images, and computed tomography data," Med. Phys. 23, 389-399 (1996).

${ }^{88}$ M. J. Murphy, "An automatic six-degree-of-freedom image registration algorithm for image-guided frameless stereotaxic radiosurgery," Med. Phys. 24, 857-866 (1997)

${ }^{89}$ A. E. Lujan, J. M. Balter, and R. K. Ten Haken, "Determination of rotations in three dimensions using two-dimensional portal image registration," Med. Phys. 25, 703-708 (1998).

${ }^{90}$ W. De Neve, F. Van den Heuvel, M. Coghe, D. Verellen, M. De Beukeleer, A. Roelstraete, P. De Roover, L. Thon, and G. Storme, "Interactive use of on-line portal imaging in pelvic radiation," Int. J. Radiat. Oncol., Biol., Phys. 25, 517-524 (1993).

${ }^{91}$ P. Bergstrom, P. O. Lofroth, and A. Widmark, "High-precision conformal radiotherapy (HPCRT) of prostate cancer-A new technique for exact positioning of the prostate at the time of treatment," Int. J. Radiat. Oncol., Biol., Phys. 42, 305-311 (1998).

${ }^{92}$ J. M. Balter, G. T. Chen, C. A. Pelizzari, S. Krishnasamy, S. Rubin, and S. Vijayakumar, "Online repositioning during treatment of the prostate: A study of potential limits and gains,'” Int. J. Radiat. Oncol., Biol., Phys. 27(1), 137-143 (1993).

${ }^{93}$ W. C. Lam, M. Partowmah, D. J. Lee, M. D. Wharam, and K. S. Lam, "On-line measurement of field placement errors in external beam radiotherapy," Br. J. Radiol. 60, 361-365 (1987).

${ }^{94}$ M. L. Graham, A. Y. Cheng, L. Y. Geer, W. R. Binns, M. W. Vannier, and J. W. Wong, "A method to analyze 2-dimensional daily radiotherapy portal images from an on-line fiber-optic imaging system,' Int. J. Radiat. Oncol., Biol., Phys. 20(3), 613-619 (1991).

${ }^{95}$ J. M. Michalski, J. W. Wong, R. L. Gerber, D. Yan, A. Cheng, M. V. Graham, M. A. Renna, P. J. Sawyer, and C. A. Perez, "The use of on-line image verification to estimate the variation in radiation therapy dose delivery,', Int. J. Radiat. Oncol., Biol., Phys. 27, 707-716 (1993). 
${ }^{96}$ A. A. el-Gayed, A. Bel, R. Vijlbrief, H. Bartelink, and J. V. Lebesque, "Time trend of patient setup deviations during pelvic irradiation using electronic portal imaging," Radiother. Oncol. 26, 162-171 (1993).

${ }^{97}$ D. A. Fein, K. P. McGee, T. E. Schultheiss, B. L. Fowble, and G. E. Hanks, "Intra- and interfractional reproducibility of tangential breast fields: A prospective on-line portal imaging study," Int. J. Radiat. Oncol., Biol., Phys. 34, 733-740 (1996).

${ }^{98}$ M. G. Herman, K. O. Khadivi, I. Gage, L. Kleinberg, and R. A. Abrams, "Effects of respiration on target and critical structure positions during treatment assessed with movie-loop electronic portal imaging," Int. J. Radiat. Oncol., Biol., Phys. 39, 163 (1997).

${ }^{99}$ E. Vigneault, J. Pouliot, J. Laverdiere, J. Roy, and M. Dorion, "Electronic portal imaging device detection of radioopaque markers for the evaluation of prostate position during megavoltage irradiation: A clinical study,"' Int. J. Radiat. Oncol., Biol., Phys. 37, 205-212 (1997).

${ }^{100}$ A. Tinger, J. M. Michalski, W. R. Bosch, R. K. Valicenti, D. A. Low, and R. J. Myerson, "An analysis of intratreatment and intertreatment displacements in pelvic radiotherapy using electronic portal imaging," Int. J. Radiat. Oncol., Biol., Phys. 34, 683-690 (1996).

${ }^{101}$ D. Yan, J. W. Wong, G. Gustafson, and A. Martinez, "A new model for 'accept or reject' strategies in off-line and on-line megavoltage treatment evaluation,'” Int. J. Radiat. Oncol., Biol., Phys. 31, 943-952 (1995).

${ }^{102}$ D. Yan, J. Wong, F. Vicini, J. Michalski, C. Pan, A. Frazier, E. Horwitz, and A. Martinez, "Adaptive modification of treatment planning to minimize the deleterious effects of treatment setup errors," Int. J. Radiat. Oncol., Biol., Phys. 38, 197-206 (1997)

${ }^{103}$ J. W. Denham, M. J. Dally, K. Hunter, J. Wheat, P. P. Fahey, and C. S. Hamilton, "Objective decision-making following a portal film: The results of a pilot study," Int. J. Radiat. Oncol., Biol., Phys. 26, 869-876 (1993).

${ }^{104}$ C. D. Mubata, A. M. Bidmead, L. M. Ellingham, V. Thompson, and D. P. Dearnaley, "Portal imaging protocol for radical dose-escalated radiotherapy treatment of prostate cancer," Int. J. Radiat. Oncol., Biol., Phys. 40, 221-231 (1998).

${ }^{105}$ K. Luchka, D. Chen, S. Shalev, G. Gluhchev, and R. Rajapakshe, "Assessing radiation and light field congruence with a video based electronic portal imaging device," Med. Phys. 23, 1245-1252 (1996).

${ }^{106}$ L. Dong, A. Shiu, S. Tung, and A. Boyer, "Verification of radiosurgery target point alignment with an electronic portal imaging device (EPID),"' Med. Phys. 24, 263-267 (1997).

${ }^{107}$ P. McGhee, T. Chu, K. Leszczynski, and P. Dunscombe, "The Siemens virtual wedge," Med. Dosim. 22, 39-41 (1997).

${ }^{108}$ J. M. Balter, D. L. McShan, J. J. Kim, and B. A. Fraass, "Automated verification of dynamic multileaf configuration using a megavoltage imager and a computer controlled radiotherapy system," Med. Phys. 24, 1002 (1997)

${ }^{109}$ M. Partridge, P. M. Evans, A. Mosleh-Shirazi, and D. Convery, "Independent verification using portal imaging of intensity-modulated beam delivery by the dynamic MLC technique," Med. Phys. 25(10), 18721879 (1998)

${ }^{110}$ F. F. Yin, M. C. Schell, and P. Rubin, "A technique of automating compensator design for lung inhomogeneity correction using an electron portal imaging device," Med. Phys. 21, 1729-1732 (1994).

${ }^{111}$ P. M. Evans, V. N. Hansen, W. P. Mayles, W. Swindell, M. Torr, and J. R. Yarnold, "Design of compensators for breast radiotherapy using electronic portal imaging," Radiother. Oncol. 37, 43-54 (1995).

${ }^{112} \mathrm{D}$. A. Low, Z. Li, and E. E. Klein, "Verification of milled twodimensional photon compensating filters using an electronic portal imaging device,"' Med. Phys. 23(6), 929-938 (1996).

${ }^{113}$ M. C. Kirby and P. C. Williams, "The use of an electronic portal imaging device for exit dosimetry and quality control measurements," Int. J. Radiat. Oncol., Biol., Phys. 31, 593-603 (1995).

${ }^{114}$ B. J. Heijmen, K. L. Pasma, M. Kroonwijk, V. G. Althof, J. C. de Boer, A. G. Visser, and H. Huizenga, "Portal dose measurement in radiotherapy using an electronic portal imaging device (EPID)," Phys. Med. Biol. 40, 1943-1955 (1995).

${ }^{115}$ M. Essers, R. Boellaard, M. van Herk, H. Lanson, and B. Mijnheer, "Transmission dosimetry with a liquid-filled electronic portal imaging device,’ Int. J. Radiat. Oncol., Biol., Phys. 34, 931-941 (1996).

${ }^{116}$ V. N. Hansen, P. M. Evans, and W. Swindell, "The application of transit dosimetry to precision radiotherapy," Med. Phys. 23, 713-721 (1996).

${ }^{117}$ T. R. McNutt, T. R. Mackie, P. Reckwerdt, N. Papanikolaou, and B. R.
Paliwal, "Calculation of portal dose using the convolution/superposition method," Med. Phys. 23, 527-535 (1996)

${ }^{118}$ R. Boellaard, M. van Herk, and B. J. Mijnheer, "A convolution model to convert transmission dose images to exit dose distributions," Med. Phys. 24, 189-199 (1997).

${ }^{119}$ K. Kesteloot, A. Dutreix, and E. van der Schueren, "A model for calculating the costs of in vivo dosimetry and portal imaging in radiotherapy departments,"' Radiother. Oncol. 28, 108-117 (1993).

${ }^{120}$ L. E. Antonuk, J. Boudry, Y. el-Mohri, W. Huang, J. H. Siewerdsen, J. Yorkston, and R. A. Street, "Large area, flat panel, amorphous silicon imagers," Proc. SPIE 2432, 216-227 (1995).

${ }^{121}$ L. E. Antonuk, J. Yorkston, W. Huang, H. Sandler, J. H. Siewerdsen, and Y. el-Mohri, "Megavoltage imaging with a large-area, flat-panel, amorphous silicon imager," Int. J. Radiat. Oncol., Biol., Phys. 36, 661-672 (1996).

${ }^{122}$ L. E. Antonuk, Y. el-Mohri, J. H. Siewerdsen, J. Yorkston, W. Huang, V. E. Scarpine, M. Verma, K. W. Jee, M. Maolinbay, and R. A. Street, "Development of a high resolution active matrix, flat-panel imager with enhanced fill factor," Proc. SPIE 3032, 2-13 (1997).

${ }^{123}$ T. Falco and B. G. Fallone, "Portal imaging: Comparing metal-plate/film with electrostatic-based detectors," Proceedings of COMP/CCPM 42nd Annual Meeting, 1996, pp. 56-59.

${ }^{124}$ D. G. Drake, D. A. Jaffray, and J. W. Wong, "Prototype amorphous silicon array based radiotherapy portal imager," Proc. SPIE 3032, 32-41 (1997).

${ }^{125}$ J. Yorkston, L. E. Antonuk, N. Seraji, W. Huang, J. H. Siewerdsen, and Y. El-Mohri, "Evaluation of the MTF for $a$-Si:H imaging arrays," Proc. SPIE 2163, 141-149 (1994).

${ }^{126}$ J. Yorkston, L. E. Antonuk, N. Seraji, W. Huang, J. H. Siewerdsen, and Y. El-Mohri, "MTF measurements with high resolution $a$-Si:H imaging arrays," Proc. SPIE 2432, 260-269 (1995).

${ }^{127}$ J. R. Earnhart and E. L. Chaney, "Modulation transfer function for a large-area amorphous silicon image receptor," Phys. Med. Biol. 42, 2531-2540 (1997)

${ }^{128}$ H. V. Culbert, R. Stagowsky, J. Ovadia, and C. Evans, "Diagnostic and therapy port verifications on the same film using an accelerator mounted diagnostic x-ray tube," Int. J. Radiat. Oncol., Biol., Phys. 10, 180 (1984).

${ }^{129}$ P. J. Biggs, M. Goitein, and M. D. Russell, "A diagnostic x ray field verification device for a 10 MV linear accelerator," Int. J. Radiat. Oncol., Biol., Phys. 11, 635-643 (1985).

${ }^{130}$ A. S. Shiu, K. R. Hogstrom, N. A. Janjan, R. S. Fields, and L. J. Peters, "Technique for verifying treatment fields using portal images with diagnostic quality,” Int. J. Radiat. Oncol., Biol., Phys. 13, 1589-1594 (1987).

${ }^{131}$ R. K. Ten Haken, K. Lam, J. M. Balter, J. Schewe, D. Hornick, J. Hetrick, S. Kota, and D. L. McShan, "Daily patient repositioning using room-based digital diagnostic xray imaging and computerized treatment table with six degrees of freedom," Fourth International Workshop on Electronic Portal Imaging, 1996, p. 42

${ }^{132}$ L. S. Johnson, S. W. Hadley, B. D. Milliken, C. A. Pelizzari, D. J. Haraf, A. Nguyen, and G. T. Y. Chen, "Initial clinical experience with an interactive, video-based patient-positioning system for head and neck treatment," Int. J. Radiat. Oncol., Biol., Phys. 36, 204 (1996).

${ }^{133}$ B. D. Milliken, S. J. Rubin, R. J. Hamilton, L. S. Johnson, and G. T. Chen, "Performance of a video-image-subtraction-based patient positioning system,'” Int. J. Radiat. Oncol., Biol., Phys. 38, 855-866 (1997).

${ }^{134}$ E. J. Holupka, I. D. Kaplan, E. C. Burdette, and G. K. Svensson, "Ultrasound image fusion for external beam radiotherapy for prostate cancer," Int. J. Radiat. Oncol., Biol., Phys. 35, 975-984 (1996).

${ }^{135}$ J. Troccaz, Y. Menguy, M. Bolla, P. Cinquin, P. Vassal, N. Laieb, L. Desbat, A. Dusserre, and S. Dal Soglio, "Conformal external radiotherapy of prostatic carcinoma: Requirements and experimental results," Radiother. Oncol. 29, 176-183 (1993).

${ }^{136}$ Y. Menguy, P. Cinquin, N. Laieb, J. Troccaz, M. Bolla, P. Vassal, A. Dusserre, and S. D. Soglio, "Optimization in conformal therapy for prostatic cancer," Proceedings of the XI International Conference on the Use of Computers in Radiotherapy, 1994, pp. 340, 341.

${ }^{137}$ J. Troccaz, N. Laieb, P. Vassal, Y. Menguy, P. Cinquin, M. Bolla, and J. Y. Giraud, "Patient setup optimization for external conformal radiotherapy,"' J. Image Guid. Surg. 1, 113-20 (1995).

${ }^{138}$ R. C. Campbell, M. P. Carol, B. H. Curran, R. E. Huber, R. V. Nash, W. D. Richard, and B. Rosen, "The use of position sensing, ultrasound imaging, and dose distribution information for improving treatment room setup accuracy,"' Med. Phys. 24, 1032 (1997). 